\title{
An error prediction framework for interferometric SAR data
}

\author{
Mohr, Johan Jacob; Merryman Boncori, John Peter
}

Published in:

I E E E Transactions on Geoscience and Remote Sensing

Link to article, DOI:

10.1109/TGRS.2008.916213

Publication date:

2008

Document Version

Publisher's PDF, also known as Version of record

Link back to DTU Orbit

Citation (APA):

Mohr, J. J., \& Merryman Boncori, J. P. (2008). An error prediction framework for interferometric SAR data. I E E E Transactions on Geoscience and Remote Sensing, 46(6), 1600-1613.

https://doi.org/10.1109/TGRS.2008.916213

\section{General rights}

Copyright and moral rights for the publications made accessible in the public portal are retained by the authors and/or other copyright owners and it is a condition of accessing publications that users recognise and abide by the legal requirements associated with these rights.

- Users may download and print one copy of any publication from the public portal for the purpose of private study or research.

- You may not further distribute the material or use it for any profit-making activity or commercial gain

- You may freely distribute the URL identifying the publication in the public portal

If you believe that this document breaches copyright please contact us providing details, and we will remove access to the work immediately and investigate your claim 


\title{
An Error Prediction Framework for Interferometric SAR Data
}

\author{
Johan Jacob Mohr and John Peter Merryman Boncori
}

\begin{abstract}
Three of the major error sources in interferometric synthetic aperture radar measurements of terrain elevation and displacement are baseline errors, atmospheric path length errors, and phase unwrapping errors. In many processing schemes, these errors are calibrated out by using ground control points (GCPs) (or an external digital elevation model). In this paper, a simple framework for the prediction of error standard deviation is outlined and investigated. Inputs are GCP position, a priori GCP accuracy, baseline calibration method along with a closed-form model for the covariance of atmospheric path length disturbances, and a model for phase unwrapping errors. The procedure can be implemented as a stand-alone add-on to standard interferometric processors. It is validated by using a set of single-frame interferograms acquired over Rome, Italy, and a double difference data set over Flevoland, The Netherlands.
\end{abstract}

Index Terms-Atmospheric disturbances, baseline error, calibration, interferometry, phase unwrapping error, synthetic aperture radar (SAR), systematic errors.

\section{INTRODUCTION}

$\mathbf{F}$ OR USERS of interferometric radar measurements of terrain elevation and displacement, estimates of accuracy are important. The basic error source is atmospheric path length disturbances. When many interferograms of the area of interest are available, the permanent scatterer technique [1] can be used to reach very good accuracies with associated error estimates by using assumptions of atmospheric statistics (low temporal correlation and high spatial correlation) and displacement statistics (high temporal correlation and low spatial correlation).

The topic of this paper, though, is focused on cases where only a few interferograms are available, e.g., due to the dynamics of the phenonema of interest (summer/winter velocities of glaciers), due to processing limitations (large area mapping), or even data availability limitation as is often the case in arctic areas. Here, classical radar interferometric techniques, typically including baseline calibration and phase unwrapping, must be used.

In these cases, a priori accuracy estimates may also be needed during product generation to ensure a proper mosaicking [2]. Attempts to quantify the errors are sparse (see, for example, [3] and [4]), but none of these take into account the correlation introduced by the use of ground control points

Manuscript received November 9, 2007; revised November 21, 2007.

J. J. Mohr is with the Department of Electrical Engineering, Technical University of Denmark, 2800 Kgs. Lyngby, Denmark (e-mail: jm@elektro.dtu.dk).

J. P. Merryman Boncori is with the National Space Institute, Technical University of Denmark, 2800 Kgs. Lyngby, Denmark (e-mail: jme@space.dtu.dk).

Digital Object Identifier 10.1109/TGRS.2008.916213
(GCPs). When a few GCPs are available, as is often the case for glacier applications, key characteristics of the baseline calibrated products are that errors depend on GCP position and strongly depend on distance to GCPs. Alternatively, precision orbit data could be used, and a reference phase could be estimated. For single-frame images, this may lead to acceptable, and quantifiable, accuracies for elevation measurements if large baselines are used [5, p. 128], but for displacement measurements, accuracies would most often be too poor.

In this paper, an approach to provide a priori error estimates of elevation and displacement accuracy is presented. An estimation procedure is outlined, and simple models are provided to demonstrate its functionality.

Even the simple models presented here, in our opinion, improve the error prediction compared with the schemes only accounting for loss of coherence and GCP uncertainty. The framework, however, allows for the substitution of the error models should new algorithms or additional information to tune them become available, e.g., on the atmospheric states at the time of acquisitions.

The method was tested on a number of data sets, and results from three are presented and discussed. It is noted that even though the single-interferogram case is investigated in a digital elevation model (DEM) generation context, the framework applies equally well to the single-interferogram displacement measurement case, for which the necessary equations are reported.

\section{ERROR MOdels}

The unwrapped interferometric phase $\varphi(x, y)$ is assumed to be composed of the following terms:

$$
\varphi(x, y)=\varphi_{\mathrm{topo}}+\varphi_{\mathrm{disp}}+\varphi_{\mathrm{base}}+\varphi_{\mathrm{atmo}}+\varphi_{\text {noise }}+\varphi_{\mathrm{unw}} .
$$

For clarity, the image coordinate $(x, y)$ dependence has been left out on all the terms on the right-hand side. In sequence, the terms represent terrain topography, terrain displacement, baseline error, atmospheric path length delay, thermal noise, and phase unwrapping error.

The topography term includes the flat-Earth component as well as the additional phase caused by the elevation above the reference surface. In principle, this definition introduces a coupling between the baseline and elevation errors. However, we assume here that if elevation is known, $\varphi_{\text {topo }}$ can be calculated and that $\varphi_{\text {base }}$ does not depend on terrain elevation. 
Note that a repeat-pass configuration is assumed. This implies that an interferometric phase (in radians) $\varphi$ corresponds to a slant range difference (in meters) $\delta$ of

$$
\delta=-\frac{\lambda}{4 \pi} \varphi
$$

where $\lambda$ denotes wavelength, and the minus sign is ignored in the error analysis. In the remaining part of this paper, $\delta$ will be denoted as "interferometric path length."

Usually, the terms of interest in (1) are $\varphi_{\text {topo }}$ and/or $\varphi_{\text {disp }}$, whereas $\varphi_{\text {base }}, \varphi_{\text {unw }}, \varphi_{\text {atmo }}$, and $\varphi_{\text {noise }}$ are regarded as unwanted noise. The usual procedure, therefore, is to eliminate or minimize the noise terms and then attribute the remaining part of the interferometric phase to topography and/or deformation.

For our error analysis, it is sufficient to assume that there is a linear relation between an error in a measured interferometric path length $\delta_{\text {topo }}$ and the inferred elevation $h$. For the singleinterferogram case, the relation is

$$
\sigma_{h} \approx\left(R \sin \theta / B_{\perp}\right) \sigma_{\delta, \text { topo }}
$$

where $R$ is the slant range, $\theta$ is the nominal angle of incidence, $B_{\perp}$ is the perpendicular baseline, $\sigma_{h}$ is the elevation standard deviation, and $\sigma_{\delta}$ is the standard deviation of the interferometric path length. The corresponding displacement error relation simply is

$$
\sigma_{\mathrm{d}} \approx \sigma_{\delta, \operatorname{disp}}
$$

where $\sigma_{\mathrm{d}}$ denotes the line-of-sight displacement standard deviation. Relations (3) and (4) are linear also in the doubledifference case (Section III-E).

For the error analysis, we can disregard the $\varphi_{\text {topo }}$ and $\varphi_{\text {disp }}$ terms in (1), which expressed in interferometric path lengh becomes

$$
\delta(x, y)=\delta_{\text {base }}+\delta_{\text {atmo }}+\delta_{\text {noise }}+\delta_{\text {unw }} .
$$

Any deviations of $\delta(x, y)$ from zero will translate into geophysical measurement errors through the linear relations previously described. A model for each of the four components is provided next.

\section{A. Baseline Errors $\left(\delta_{\text {base }}\right)$}

In this paper, only linear baseline errors will be considered. Following the procedure of [4], a linear baseline variation in azimuth is approximated by a path length variation of the form

$$
\begin{aligned}
\delta_{\text {base }}(x, y) & =b_{1}+b_{2} x+b_{3} y+b_{4} x y \\
& =\boldsymbol{p} \cdot \boldsymbol{b}
\end{aligned}
$$

where $\boldsymbol{p}=[1, x, y, x y]^{\prime}, \boldsymbol{b}=\left[b_{1}, b_{2}, b_{3}, b_{4}\right]^{\prime}, x$ is the alongtrack coordinate, $y$ is the across-track coordinate, and $b_{i}$ 's are the constants to be determined by baseline calibration.

\section{B. Atmospheric Path Length Variations $\left(\delta_{\text {atmo }}\right)$}

Synthetic aperture radar (SAR) interferometers are known to be sensitive to temporal variations in the spatial distribution of water vapor in the troposphere [6]-[9] and of free electron density in the ionosphere [10]-[12]. Furthermore, for scenes encompassing significant elevation differences, changes in the vertical profile of the refractive index may also cause nonnegligible artifacts [5], [13].

In this paragraph, only water-vapor fluctuations shall be considered. The error-estimation framework of this paper makes use of the second-order statistics of error sources, as detailed in Section III. Concerning water-vapor-induced delay fluctuations, several models based on turbulence theory have been developed within the radio propagation [17], atmospheric science [18], very long baseline interferometry [14], GlobalPositioning-System (GPS) [13], and SAR [5] communities over the past 30 years. These were validated against independent measurement techniques and, nevertheless, share important common features, particularly a zenith-delay structure function with a power index mainly in the $[2 / 3,5 / 3]$ interval for typical SAR scales (from 100 to $400 \mathrm{~km}$ ) and a correlation length in the [700 km, $3000 \mathrm{~km}$ ] range (this does not apply for [5] in which shorter distances were considered). To demonstrate the impact on the error prediction framework of including atmospheric delay among the error sources, we shall make use of the model derived in [16] and reported in the Appendix. This is based on and in agreement with all the aforementioned studies and shares the hypotheses of wide-sense stationarity and circular symmetry of the delay. We prefer it here because it provides a closed-form expression for the second-order delay statistics and a tunable scale factor.

In the following, results useful for the error-estimation framework of this paper shall be recalled, assuming widesense stationarity and circular symmetry for the delay statistics, although these assumptions are not strictly required by the framework.

In [15], delay is discussed in a GPS context, where one-way zenith delay (in meters) $\tau$ is the basic quantity. Delay at offnadir angles and zenith delay are proportional, and for a planeparallel refractive medium, the scaling factor is stated to be

$$
m(\theta)=\frac{1}{\cos \theta}
$$

where $\theta$ is the off-nadir angle, i.e., approximately the nominal angle of incidence on ground. It is also noted that with the chosen definition (2), one-way delays should not be multiplied by two when interferometric path lengths are calculated (see also [15, eqs. (10) and (11)]).

According to Williams et al. [15], the covariance between zenith delays at two points $(i, j)$ separated in space and/or time can be calculated by using

$$
\operatorname{Cov}\left\{\tau_{i}, \tau_{j}\right\}=\frac{1}{2}(D(\infty)-D(R))
$$

where

$$
R^{2}=\left|\overrightarrow{r_{i}}-\overrightarrow{r_{j}}\right|^{2}+\left|s\left(T_{i}-T_{j}\right)\right|^{2}
$$


In (8b), $\vec{r}$ represents the point positions, $T$ represents the times of interest, and $s$ represents the wind speed at a reference tropospheric height. In [16], the zenith-delay structure function $D(R)$ is approximated by

$$
D(R)=P_{0} C_{0}\left[\frac{C_{1} I_{1}\left(\frac{R}{h}\right) R^{2 / 3}}{1+\left(\frac{R}{L}\right)^{2 / 3}}+C_{2} I_{2}\left(\frac{R}{h}\right) R^{5 / 3}\right] .
$$

The values of the constants $P_{0}, L, h, C_{0}, C_{1}$, and $C_{2}$ and the expressions of $I_{1}(\cdot)$ and $I_{2}(\cdot)$, which are the polynomial functions of $R / h$, are reported in the Appendix. For the globally representative atmospheric statistics chosen in [16], $D(\infty)=$ $11.52 \mathrm{~cm}^{2}$, and it is also noted that $D(0)=0$.

By using (8a), the variance of the interferometric path length $\delta_{i}=m(\theta)\left(\tau_{2, i}-\tau_{1, i}\right)$ in one pixel $i$, in one interferogram formed from the Single Look Complex (SLC) images 1 and 2 , is found to be

$$
\begin{aligned}
\operatorname{Var}\left\{\delta_{i}\right\} & =m^{2}(\theta) \operatorname{Var}\left\{\tau_{2, i}-\tau_{1, i}\right\} \\
& =m^{2}(\theta)\left(\operatorname{Var}\left\{\tau_{1, i}\right\}+\operatorname{Var}\left\{\tau_{2, i}\right\}-2 \operatorname{Cov}\left\{\tau_{2, i}, \tau_{1, i}\right\}\right) \\
& \approx m^{2}(\theta)\left(\frac{1}{2} D(\infty)+\frac{1}{2} D(\infty)-2 \cdot 0\right) \\
& =m^{2}(\theta) D(\infty) \\
& =\sigma_{\text {tropo }}^{2} .
\end{aligned}
$$

In the aforementioned equation, a time separation between SLC acquisitions of a day or more is assumed, ensuring that the distance (8b) between one pixel in the two SLCs is very large even with an $s$ having a value of a few meters per second.

Similarly, assuming a statistically similar atmospheric state at the two acquisitions, the covariance between interferometric path lengths at two points $(i, j)$ in one interferogram, which are separated by the distance $r$, is found to be

$$
\begin{aligned}
\operatorname{Cov}\left\{\delta_{i}, \delta_{j}\right\} & =m^{2}(\theta)(D(\infty)-D(r)) \\
& =C_{\text {tropo }}(r)
\end{aligned}
$$

which states that the atmospheric interferometric path length at two closely separated interferogram pixels has a high covariance which decreases toward zero as the pixel distance increases. The variance of the difference between interferometric path lengths at two points $(i, j)$ in one interferogram is found to be

$$
\operatorname{Var}\left\{\delta_{i}-\delta_{j}\right\}=2 m^{2}(\theta) D(r)
$$

\section{Noise $\left(\delta_{\text {noise }}\right)$}

We use the standard formula for phase noise

$$
\sigma_{\varphi}=\frac{1}{\sqrt{2 N}} \frac{\sqrt{1-\gamma^{2}}}{\gamma}
$$

where $N$ is the number of looks, and $\gamma$ is the correlation coefficient [19]. Phase noise is converted to interferometric path length by using (2). In many situations, though, noise has a small impact on the final accuracy, implying that the use of a single average value for all pixels in the entire image may suffice.

\section{Phase Unwrapping Errors $\left(\delta_{\mathrm{unw}}\right)$}

Unwrapping errors are known to be caused by phase noise, phase undersampling, and phase discontinuities [20]. Phase noise appears in areas with temporal decorrelation, low SNR, and radar shadow [21]. Phase undersampling occurs when the phase gradient is greater than $\pi$ in magnitude due to the underlying topography or due to displacement gradients. Phase noise, however, causes undersampling to also occur at lower gradients [20], [21]. Finally, phase discontinuities are due to discontinuous surface deformation (e.g., at sliding faults or at glacier-rock interfaces) and to radar layover [20], [22].

Local indicators of potentially critical unwrapping situations are known to be residue density, coherence, wrapped phase gradient, image intensity, and estimated slope. Some of these are strongly correlated, namely, residue density and coherence [23], [24] and intensity and topographic slope [25]. Errors may, however, also propagate into locally noncritical regions, during phase-gradient integration, leading to medium- and large-scale errors.

For the framework of this paper, a model of the covariance of phase unwrapping errors for any pair of interferogram pixels is required. This problem is split into two subproblems. The first is to obtain a segmentation mask of consistently unwrapped areas. The second is to assign variance and covariance values to points belonging to the same region and to different regions.

Two procedures can be found in the literature to automatically produce a map of reliably unwrapped areas, namely, [26] and [27]. Both account for scene-specific parameters, namely, residue density and coherence, respectively, as well as algorithm-specific ones, i.e., the position of branch cuts. We chose to test the application of the method of [26]. In this procedure, the unwrapped phase field is needed as input. Residue density is estimated on an $N_{\mathrm{r}} \times N_{\mathrm{r}}$ window. Pixels exceeding a threshold $t_{\mathrm{s}}$ are assigned to a masked-out region, and the remaining ones are assigned to a valid one. Holes smaller than a specified size $N_{\mathrm{h}}$ are filled in both regions. Second, pixels neighboring phase jumps greater than $\pi$ (branch cuts) are assigned to the masked-out region. Finally, the valid pixels are divided into connected regions, allegedly the consistently unwrapped ones, creating a segmentation mask.

For the application at hand, we added two steps to the algorithm, which are aimed at improving the identification of medium- to large-scale segments. Before connected regions are identified, we perform a binary erosion of the valid region by using a square structure element of size $N_{\mathrm{e}} \times N_{\mathrm{e}}$. We then identify connected regions and perform a binary dilation of each connected region by using a square structure element of size $N_{\mathrm{d}} \times N_{\mathrm{d}}$. The purpose of the erosion is to join branch cuts to residue-dense areas if these lie within a range of $N_{\mathrm{e}}$ pixels from each other. The dilation which follows is carried out with $N_{\mathrm{d}} \geq N_{\mathrm{e}}$ and is used to compensate for the erosion, particularly at the border of water bodies and decorrelated pixels, and to fill holes in each segment of size $N_{\mathrm{d}}-N_{\mathrm{e}}$. 
The derived segmentation mask, denoted by $\operatorname{seg}(\boldsymbol{q})$ in the following, is used to compute error statistics for a pair of pixels, i.e., $\boldsymbol{q}_{1}=\left(x_{1}, y_{1}\right)$ and $\boldsymbol{q}_{2}=\left(x_{2}, y_{2}\right)$. It is assumed that the values in $\operatorname{seg}(\boldsymbol{q})$ are integers, with zero denoting the maskedout region and positive values denoting the connected segments. The rationale is that if $\boldsymbol{q}_{1}$ and $\boldsymbol{q}_{2}$ belong to the same segment, their phase unwrapping error will be highly (maximally) correlated; otherwise, they are assumed to be decorrelated. Denoting with $C_{12}$ the covariance between the phase unwrapping error at $\boldsymbol{q}_{1}$ and $\boldsymbol{q}_{2}, \max \left(C_{12}\right)=\sigma_{1} \sigma_{2}$, where $\sigma_{1}$ and $\sigma_{2}$ are the standard deviations of the phase unwrapping error at $\boldsymbol{q}_{1}$ and $\boldsymbol{q}_{2}$. These values are unknown however. Without any further knowledge, the single-cycle error variance is assumed, i.e., $\sigma_{1}=\sigma_{2}=\sigma_{s}=\left(\left(0+(2 \pi)^{2}+(-2 \pi)^{2}\right) / 3\right)^{1 / 2}=$ $0.82 \cdot 2 \pi$. If $\boldsymbol{q}_{1}$ and $\boldsymbol{q}_{2}$ belong to different segments, or if one falls in the masked-out region (where local errors might occur), we do not know anything about $C_{12}$. We state this ignorance by setting $C_{12}=0$, although this may not always be the case (probably, two adjacent segments have actually a correlated phase unwrapping error since error might have propagated from one region into the other). Therefore, we write

$$
C_{12}= \begin{cases}\sigma_{\mathrm{s}}^{2}, & \boldsymbol{q}_{1}=\boldsymbol{q}_{2} \\ \sigma_{\mathrm{s}}^{2}, & \operatorname{seg}\left(\boldsymbol{q}_{1}\right)=\operatorname{seg}\left(\boldsymbol{q}_{2}\right) \wedge \operatorname{seg}\left(\boldsymbol{q}_{1}\right) \operatorname{seg}\left(\boldsymbol{q}_{2}\right) \neq 0 \\ 0, & \text { otherwise. }\end{cases}
$$

For the test cases described in Section IV, we set $N_{\mathrm{r}}=7$, $N_{\mathrm{h}}=20, t_{\mathrm{s}}=0.05, N_{\mathrm{e}}=3$, and $N_{\mathrm{d}}=13$. As discussed in Section V-B and in the conclusion, a finer tuning of these parameters may lead to an improved performance.

\section{DeVEloped Algorithm}

\section{A. Baseline Calibration and Absolute Phase}

Usually, the interferometric path length has to be calibrated before it is used to derive elevation and/or displacement. A common approach is to attribute all (slow-varying) errors to baseline uncertainties, which are estimated by using GCPs, with known elevation and displacement (often equal to zero). For each GCP, the expected topographic and displacement interferometric path lengths are calculated and subtracted from the measured path length. The resulting interferometric path length $\delta_{\text {obs }}$ is then composed of the following terms:

$$
\delta_{\text {obs }}=\delta_{\text {base }}+\left(\delta_{\text {atmo }}+\delta_{\text {noise }}+\delta_{\text {unw }}+\delta_{\text {gcp }}\right) .
$$

The first term on the right-hand side is the deterministic baseline error, which may be modeled by (6). The remaining four terms are realizations of stochastic processes with zero means. The last term $\delta_{\text {gcp }}$ represents the error of the GCP elevation and displacement terms, with standard deviations which may be calculated from

$$
\sigma_{\mathrm{gcp}}^{2}=\left(\frac{B_{\perp}}{R \sin \theta}\right)^{2} \sigma_{h}^{2}+\sigma_{\mathrm{d}}^{2}
$$

The parameter $\sigma_{\mathrm{d}}$ is typically used when the GCPs situated in terrain with displacement are used for calibration. One example could be the GCPs on ice divides, where the velocity is believed to be zero. In that case, the user should specify the accuracy of this assumption through $\sigma_{\mathrm{d}}$.

At first, it might seem problematic to annihilate the phase unwrapping constant into the baseline estimate since a constant phase (often denoted as "the absolute phase") cannot be modeled exactly by a baseline error. In practice, however, it is very difficult to distinguish an absolute phase error from a baseline error in the midswath line-of-sight direction. This is particularly true for satellite geometries having small variations in look angle from near to far range. This is also highlighted by (6), where the $b_{1}$ term is equivalent to an absolute phase term. Similarly, a constant interferometric path length caused by the atmosphere is annihilated into the baseline error. The use of the simplified baseline model thus allows (forces) us to assume a zero mean for the error terms $\delta_{\text {atmo }}, \delta_{\text {noise }}, \delta_{\text {unw }}$, and $\delta_{\text {gcp }}$. For simplicity, they are also assumed to be normally distributed and mutually independent.

It is noted that although a simplified baseline model is used for error estimation, it is recommended to use a physically based model for the actual baseline estimation. One commonly used option is a four-parameter model (offset and skew in the horizontal and vertical directions). In this case, it is also recommended to remove the bulk part of the absolute phase before the baseline calibration, leave out the absolute phase estimation, and thus let the baseline estimate account for the absolute phase.

\section{B. Baseline Solution}

With the assumptions previously outlined, the baseline-errorestimation problem with $N$ GCPs may be stated as

$$
\boldsymbol{y}=\boldsymbol{X} \boldsymbol{b}+\varepsilon
$$

where

$$
\begin{aligned}
\boldsymbol{y} & =\left[\delta_{1}, \ldots, \delta_{N}\right]^{\prime} \\
\boldsymbol{X} & =\left[\begin{array}{cccc}
1 & x_{1} & y_{1} & x_{1} y_{1} \\
\vdots & \vdots & \vdots & \vdots \\
1 & x_{N} & y_{N} & x_{N} y_{N}
\end{array}\right] \\
\boldsymbol{\varepsilon} & \in N\left(0, \boldsymbol{\Sigma}_{\varepsilon}\right) \\
\boldsymbol{\Sigma}_{\varepsilon} & =\boldsymbol{\Sigma}_{\text {atmo }}+\boldsymbol{\Sigma}_{\text {noise }}+\boldsymbol{\Sigma}_{\text {unw }}+\boldsymbol{\Sigma}_{\text {gcp }} .
\end{aligned}
$$

The standard linear least square estimate $\hat{b}$ is

$$
\begin{aligned}
\hat{\boldsymbol{b}} & =\left(\boldsymbol{X}^{\prime} \boldsymbol{\Sigma}_{\varepsilon}^{-1} \boldsymbol{X}\right)^{-1} \boldsymbol{X}^{\prime} \boldsymbol{\Sigma}_{\varepsilon}^{-1} \boldsymbol{y} \\
& =\boldsymbol{W} \boldsymbol{y}
\end{aligned}
$$

A baseline estimation using a (linearized) physically based model would use a different $\boldsymbol{X}$ and $\boldsymbol{b}$ but an identical variance-covariance matrix $\boldsymbol{\Sigma}_{\varepsilon}$.

\section{Calibrated Path Length Uncertainty}

The next step is to calculate the variance of interferometric path length $\delta_{\mathrm{p}}^{*}$ at a pixel with the coordinates $(x, y)$ after the 
baseline calibration. This section concerns the case where the simplified model (6) is actually used for the baseline calibration. In this case, the calibrated path length is $\delta_{\mathrm{p}}^{*}=\delta_{\mathrm{p}}-\boldsymbol{p} \cdot \hat{\boldsymbol{b}}$. The residual error includes errors on the observation $\delta_{\mathrm{p}}$ itself, namely, $\delta_{\mathrm{p}, \text { atmo }}, \delta_{\mathrm{p}, \text { noise }}$, and $\delta_{\mathrm{p} \text {,unw }}$, as well as errors of the baseline calibration term $\boldsymbol{p} \cdot \hat{\boldsymbol{b}}$, which is correlated with $\left(\delta_{\mathrm{p}, \text { atmo }}, \delta_{\mathrm{p} \text {,noise }}, \delta_{\mathrm{p}, \text { unw }}\right)$-particularly in the vicinity of GCPs. The variance of $\delta_{\mathrm{p}}^{*}$ is rewritten as

$$
\begin{aligned}
\operatorname{Var}\left\{\delta_{\mathrm{p}}^{*}\right\} & =\operatorname{Var}\left\{\delta_{\mathrm{p}}-\boldsymbol{p} \cdot \hat{\boldsymbol{b}}\right\} \\
& =\operatorname{Var}\left\{\delta_{\mathrm{p}}-\boldsymbol{p} \cdot \boldsymbol{W} \boldsymbol{y}\right\} \\
& =\operatorname{Var}\left\{\boldsymbol{w}_{\mathrm{p}} \cdot \boldsymbol{y}_{\mathrm{p}}\right\}
\end{aligned}
$$

where

$$
\begin{aligned}
& \boldsymbol{w}_{\mathrm{p}}=\left[1, w_{\mathrm{p}, 1}, \ldots, w_{\mathrm{p}, N}\right]^{\prime} \\
& w_{\mathrm{p}, i}=\sum_{j=1}^{4} p_{j} W_{j, i} \\
& \boldsymbol{y}_{\mathrm{p}}=\left[\delta_{\mathrm{p}},-\delta_{1}, \ldots,-\delta_{N}\right]^{\prime} \\
& \boldsymbol{y}_{\mathrm{p}} \in N\left(0, \boldsymbol{\Sigma}_{\mathrm{p}}\right) \\
& \boldsymbol{\Sigma}_{\mathrm{p}}=\left(\begin{array}{c|ccc}
V_{\mathrm{p}} & -C_{\mathrm{p}, 1} & \cdots & -C_{\mathrm{p}, N} \\
\hline-C_{\mathrm{p}, 1} & & & \\
\vdots & & \boldsymbol{\Sigma}_{\varepsilon} & \\
-C_{\mathrm{p}, N} & &
\end{array}\right) \\
& V_{\mathrm{p}}=\operatorname{Var}\left\{\delta_{\mathrm{p}}\right\}=\sigma_{\text {atmo }}^{2}+\sigma_{\text {unw }}^{2}+\sigma_{\text {noise }}^{2} \\
& C_{\mathrm{p}, i}=\operatorname{Cov}\left\{\delta_{\mathrm{p}}, \delta_{i}\right\}=C_{\mathrm{atmo}}\left(r_{\mathrm{p}, i}\right)+C_{\mathrm{unw}}\left(r_{\mathrm{p}, i}\right) \\
& r_{\mathrm{p}, i}=\left(\left(x_{i}-x_{\mathrm{p}}\right)^{2}+\left(y_{i}-y_{\mathrm{p}}\right)^{2}\right)^{1 / 2} \text {. }
\end{aligned}
$$

Now, the standard deviation $\sigma_{\mathrm{p}^{*}}$ of the calibrated single-pixel interferometric path length can be calculated from

$$
\sigma_{\mathrm{p}^{*}}^{2}=\boldsymbol{w}_{\mathrm{p}}^{\prime} \boldsymbol{\Sigma}_{\mathrm{p}} \boldsymbol{w}_{\mathrm{p}}
$$

To summarize, the following information is needed to calculate the uncertainty.

1) The weight matrix $\boldsymbol{W}$ used to calculate the baseline estimate.

2) The variance-covariance matrix $\boldsymbol{\Sigma}_{\mathrm{p}}$ for observations $\boldsymbol{y}_{\mathrm{p}}$ used. To calculate that, the position of all GCPs must be available.

Note that the baseline estimate itself $\hat{b}$ is not needed.

Either the $\boldsymbol{W}$ matrix should be output from the baseline calibration module or sufficient information about the procedure should be available, so that it can be recalculated. If $\boldsymbol{W}$ is output, the $(x, y)$ coordinate system should also be specified, whereas any $(x, y)$ coordinate system (e.g., pixel numbers) can be used if $\boldsymbol{W}$ is recalculated.

Methods for calculating the variances $(19 \mathrm{~g})$ and covariances (19h) are proposed in Sections II-B to D, but if more accurate information is somehow available, it should be used. Note that the $\Sigma_{\varepsilon}$ part of (19f) does not need to be identical to the one used for the baseline estimation. A common situation is that a unity variance-covariance matrix is used for the baseline estimation, but this should not prevent the use of a better covariance model for error prediction. Also note that sequence and $(x, y)$ positions of used GCPs are needed to calculate $\boldsymbol{\Sigma}_{\mathrm{p}}$.

\section{Height and Displacement From a Single Interferogram}

To estimate the standard deviation of the height measured from a single interferogram, that of the calibrated path length (20) can be substituted to the right-hand side of (3).

Concerning displacement measurement from a single interferogram, an external DEM is required in general [28]. Typically, in this case, the GCPs used from the baseline calibration are extracted from the available DEM. This introduces a correlation between DEM height errors and calibrated path length, for pixels colocated with an extracted GCP. By using the notation of Section III-C, the following equation can be proved:

$$
\sigma_{\mathrm{d}}= \begin{cases}\sqrt{\sigma_{\mathrm{p}^{*}}^{2}+\frac{\sigma_{h, \mathrm{DEM}}^{2}}{k_{h}^{2}}\left(1+2 w_{\mathrm{p}, i}\right)}, & \left(x_{\mathrm{p}}, y_{\mathrm{p}}\right)=\left(x_{i}, y_{i}\right) \\ \sqrt{\sigma_{\mathrm{p}^{*}}^{2}+\frac{\sigma_{h, \mathrm{DEM}}^{2}}{k_{h}^{2}},} & \text { otherwise }\end{cases}
$$

where $k_{h}=-R \sin \theta / B_{\perp}$. The DEM height standard deviation at the pixel position is represented by $\sigma_{h, \text { DEM }}$. The correlation between the DEM height error and the calibrated pixel path length depends on $w_{\mathrm{p}, i}$ defined in (19c). If several GCPs are used, this term is expected to be small since a robust calibration should not assign a high weight to any single GCP.

\section{E. Double-Difference Case}

The framework previously presented can be extended to more complicated scenarios where the elevation or displacement for a pixel $g_{\mathrm{p}}$ is derived from a linear combination of calibrated interferogram values, i.e.,

$$
g_{\mathrm{p}}=k_{1} \delta_{\mathrm{p}, 1}^{*}+k_{2} \delta_{\mathrm{p}, 2}^{*}+\cdots .
$$

Examples are differential interferometry and stacking of interferograms. Even for the simple double-difference case, though, different scenarios exist (e.g., continuous motion versus abrupt motion), leading to slightly different results, and thus, it is left to the reader to adapt the method to his particular needs.

To demonstrate the principle, the double-difference, tandem, and continuous motion case is described next. The key issue is that the $\delta_{\mathrm{gcp}}$ errors are correlated from interferogram to interferogram, leading to the surprising result that the final velocity accuracy under certain circumstances is independent of the GCP elevation accuracy.

Following the approach in [4], the temporal baseline is denoted by $T$, the perpendicular baselines by $B_{1}$ and $B_{2}$, the slant range by $R$, and the angle of incidence by $\theta$. The elevation product constants in (22) are

$$
k_{h, 1}=-\frac{R \sin \theta}{B_{1}-B_{2}} \wedge k_{h, 2}=-k_{h, 1}
$$


and the corresponding velocity product constants are

$$
k_{\mathrm{v}, 1}=\frac{-B_{2}}{T\left(B_{1}-B_{2}\right)} \wedge k_{\mathrm{v}, 2}=\frac{B_{1}}{T\left(B_{1}-B_{2}\right)} .
$$

To simplify the notation, the pixel position dependence on $\left(B_{1}, B_{2}, R, \theta\right)$ has been left out, but when the quantities are used for a specific pixel, the corresponding values should be used. An expression for the variance of (22) simply is

$$
\begin{aligned}
\operatorname{Var}\left\{g_{\mathrm{p}}\right\}= & k_{1}^{2} \boldsymbol{w}_{\mathrm{p}, 1}^{\prime} \boldsymbol{\Sigma}_{\mathrm{p}, 1} \boldsymbol{w}_{\mathrm{p}, 1}+k_{2}^{2} \boldsymbol{w}_{\mathrm{p}, 2}^{\prime} \boldsymbol{\Sigma}_{\mathrm{p}, 2} \boldsymbol{w}_{\mathrm{p}, 2} \\
& +2 k_{1} k_{2} \operatorname{Cov}\left\{\delta_{\mathrm{p}, 1}-\boldsymbol{p}_{1} \cdot \hat{\boldsymbol{b}}_{1}, \delta_{\mathrm{p}, 2}-\boldsymbol{p}_{2} \cdot \hat{\boldsymbol{b}}_{2}\right\}
\end{aligned}
$$

The first two terms can be calculated from (19). To calculate the last term, the covariances $\operatorname{Cov}\left\{y_{\mathrm{p}, 1, i}, y_{\mathrm{p}, 2, j}\right\}$ are needed. For the tandem case, it is reasonable to assume that the error on the observation $\delta_{\mathrm{p}, 1}$ in one interferogram is uncorrelated with the observation $\delta_{\mathrm{p}, 2}$ in the other. Likewise, it is reasonable to assume that the error on $\delta_{\mathrm{p}, 1}$ is uncorrelated with the errors on the pixels used for GCP calibration in the other interferogram $\delta_{2, j}$. The correlation between the atmospheric, the unwrapping, and the noise errors in pixels used for the baseline calibration in the two interferograms is also most likely uncorrelated. The errors on elevation and displacement for a GCP used in both interferograms, though, are not uncorrelated. An interesting special case is when the following conditions are achieved.

1) The same GCPs are used for both interferograms (for clarity, the same sequence is also assumed);

2) There is no uncertainty on the displacement term of the GCPs (which, in this case, are typically stationary).

In that case, the last term can be calculated from

$$
\begin{aligned}
& 2 k_{1} k_{2} \operatorname{Cov}\left\{\delta_{\mathrm{p}, 1}-\boldsymbol{p}_{1} \cdot \hat{\boldsymbol{b}}_{1}, \delta_{\mathrm{p}, 2}-\boldsymbol{p}_{2} \cdot \hat{\boldsymbol{b}}_{2}\right\} \\
&=2 k_{1} k_{2} \boldsymbol{w}_{\mathrm{p}, 1}^{\prime} \boldsymbol{\Sigma}_{\mathrm{p}, 12} \boldsymbol{w}_{\mathrm{p}, 2}
\end{aligned}
$$

where

$$
\boldsymbol{\Sigma}_{\mathrm{p}, 12}=\left(\begin{array}{c|ccc}
0 & 0 & \cdots & 0 \\
\hline 0 & \frac{B_{1} B_{2}}{(R \sin \theta)^{2}} \sigma_{h, 1}^{2} & \cdots & 0 \\
\vdots & \vdots & \ddots & \vdots \\
0 & 0 & \cdots & \frac{B_{1} B_{2}}{(R \sin \theta)^{2}} \sigma_{h, N}^{2}
\end{array}\right) .
$$

By using (23b), it is now seen that the GCP elevation errors in the last term of (25) cancel the elevation errors in the first two terms. In other words, in the special case previously outlined, the combined velocity error is independent of the accuracy of the GCP elevations. The combined velocity error, though, is still dependent on the number of GCPs and their distribution. Many and well-distributed GCPs are still advantageous in order to reduce the general influence of atmosphere, unwrapping, and noise.

\section{Demonstration And VALIDATION}

\section{A. Application to Elevation Measurement}

The framework of Section III-C was applied to predict the elevation error in two DEMs obtained from single interferometric pairs, namely, ascending European Remote Sensing 1 and 2 satellite (ERS) tandem e1_20701/e2_01028, frame 837 (referred to as $\mathrm{I7}$ in the following) and descending ERStandem e1_39646/e2_19973, frame 2763 (referred to as I5 in the following), both covering the city of Rome, Italy, and surroundings. For I7, $B_{\perp}=-50 \mathrm{~m}$, whereas $B_{\perp}=-211 \mathrm{~m}$ for I5, corresponding to heights of ambiguity of 188 and $45 \mathrm{~m}$, respectively. GCPs for the baseline calibration were extracted from an Istituto Geografico Militare DEM, with a 10-m error standard deviation. The interferometric DEMs were generated by using DTU's InSAR Postprocessing Software.

The obtained elevations were compared with a Shuttle Radar Topography Mission (SRTM) DEM of the same area, with an expected 13-m error standard deviation. In the following, errors in the SRTM DEM shall not be explicitly taken into account. In comparing height differences, it should therefore be borne in mind that (uncorrelated) disagreements of the order of magnitude of the SRTM accuracy are not significant.

The I7 test case is analyzed in Fig. 1. Fig. 1(a) plots the observed height difference with the SRTM DEM, together with the GCP configuration. The image is dominated by widespread correlated error structures, which resemble atmospheric propagation artifacts observed in other studies. In the area covered by the GCPs, there is a rapid, yet not abrupt, transition from positive to negative height differences (red to yellow), indicating a wavelike disturbance. In the top-left corner, a height difference of $-400 \mathrm{~m}$ is found.

The predicted error standard deviation accounting for all error sources is shown in Fig. 1(b). In most of the image, observations fall within two predicted standard deviations, as shown in Fig. 1(c). It can also be noted by comparing Fig. 1(c) and (d) that the main contribution to the error prediction is given by the atmospheric model. Based on coherence and GCP height uncertainty only, the predicted values significantly underestimate the observed ones in all areas of the image, as shown in Fig. 1(f). For this test case, the phase unwrapping error model predicts some local errors only, such as the one marked within a rectangle in the top-right part of Fig. 1(e) and (a). Indeed, no medium- or large-scale unwrapping error is apparent however in Fig. 1(a).

A more challenging test case for the phase unwrapping error model is provided by the I5 data, as analyzed in Fig. 2. Fig. 2(a) plots the observed height difference with the SRTM DEM, together with the GCP configuration. In this case, the image is dominated by patches of highly correlated errors, with abrupt transitions of multiples of the height of ambiguity $(45 \mathrm{~m})$. These are clearly phase unwrapping errors. The segmentation mask shown in Fig. 2(b) attempts to identify these regions, following the procedure described in Section II-D. It can be seen that it is only partially successful. It has been seen that incrementing the erosion window size allows more segments to be identified, at the price, however, of oversegmentation of already existing regions. Furthermore, erroneous 


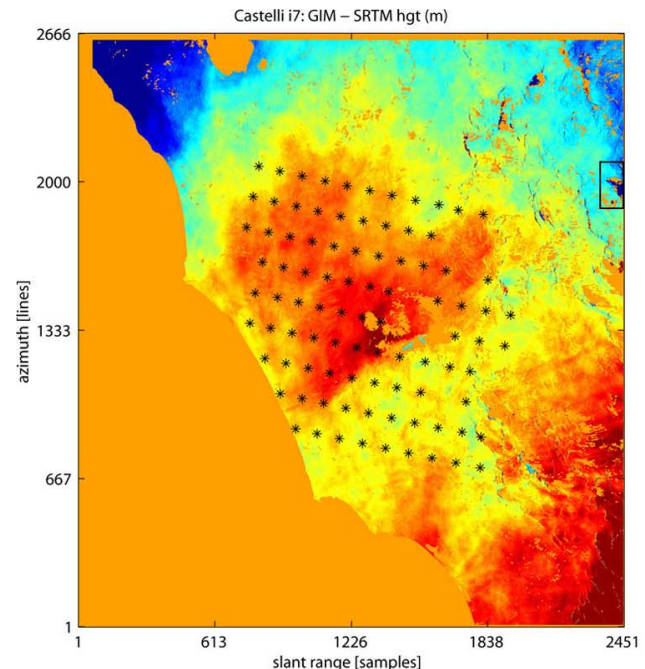

a)

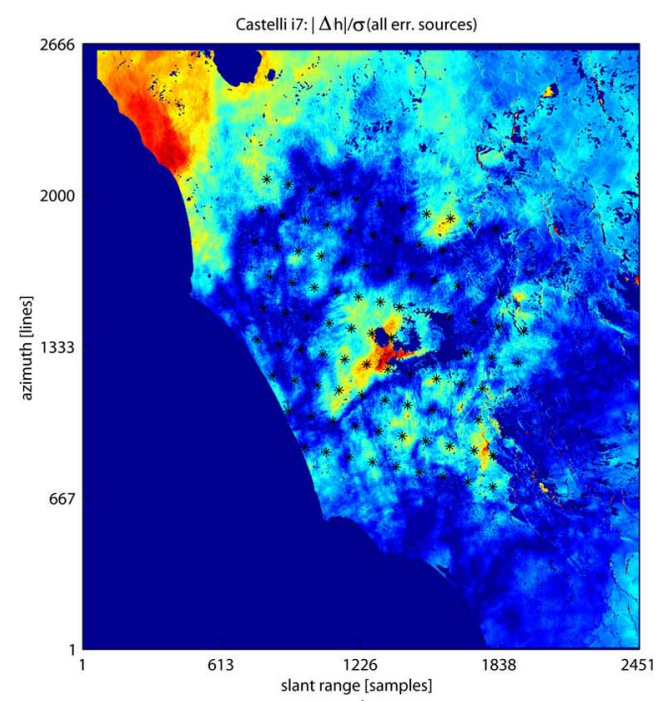

c)

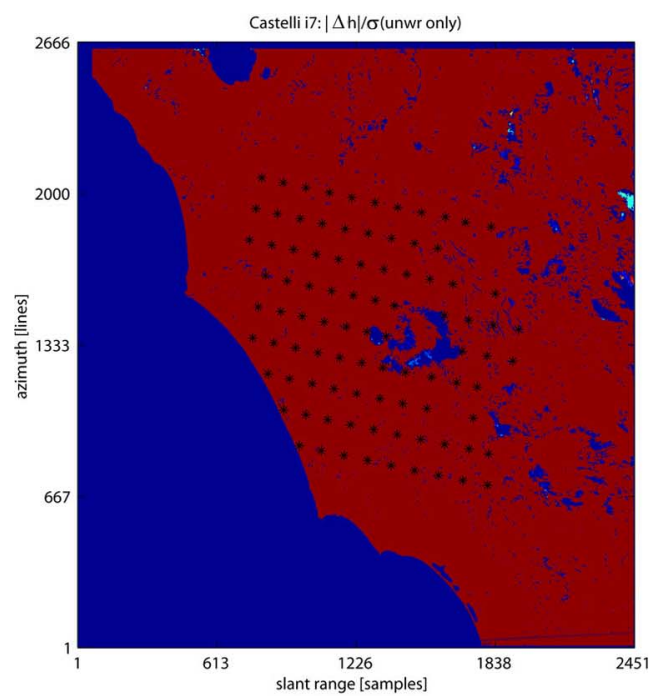

e)
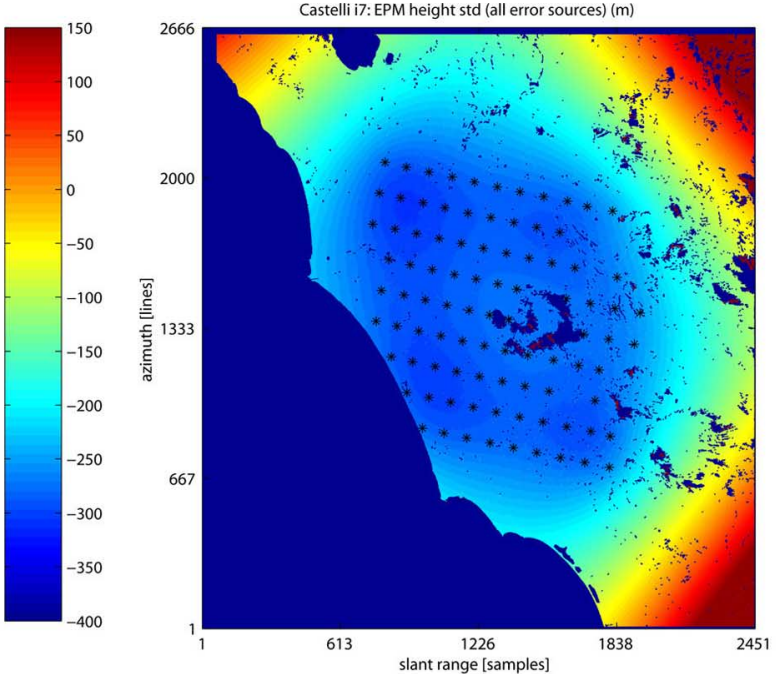

b)
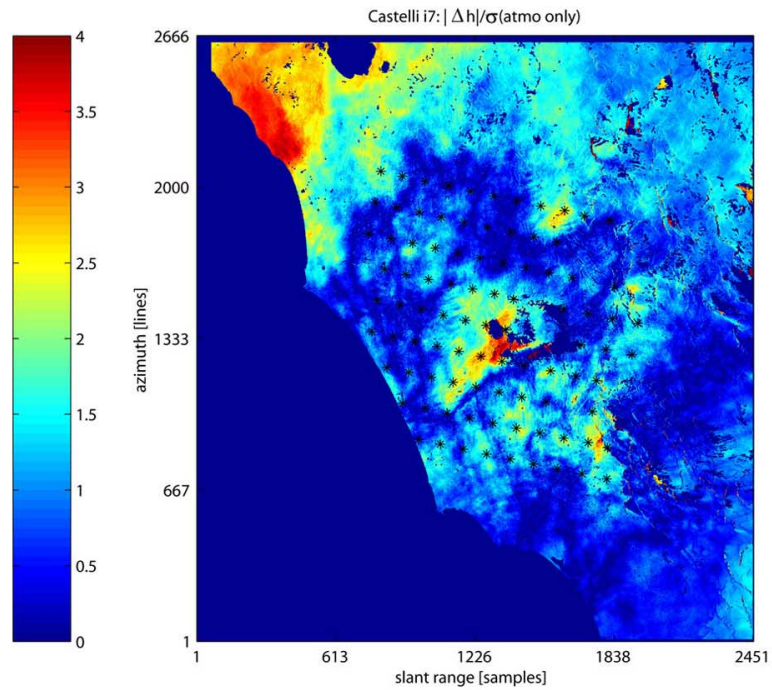

d)
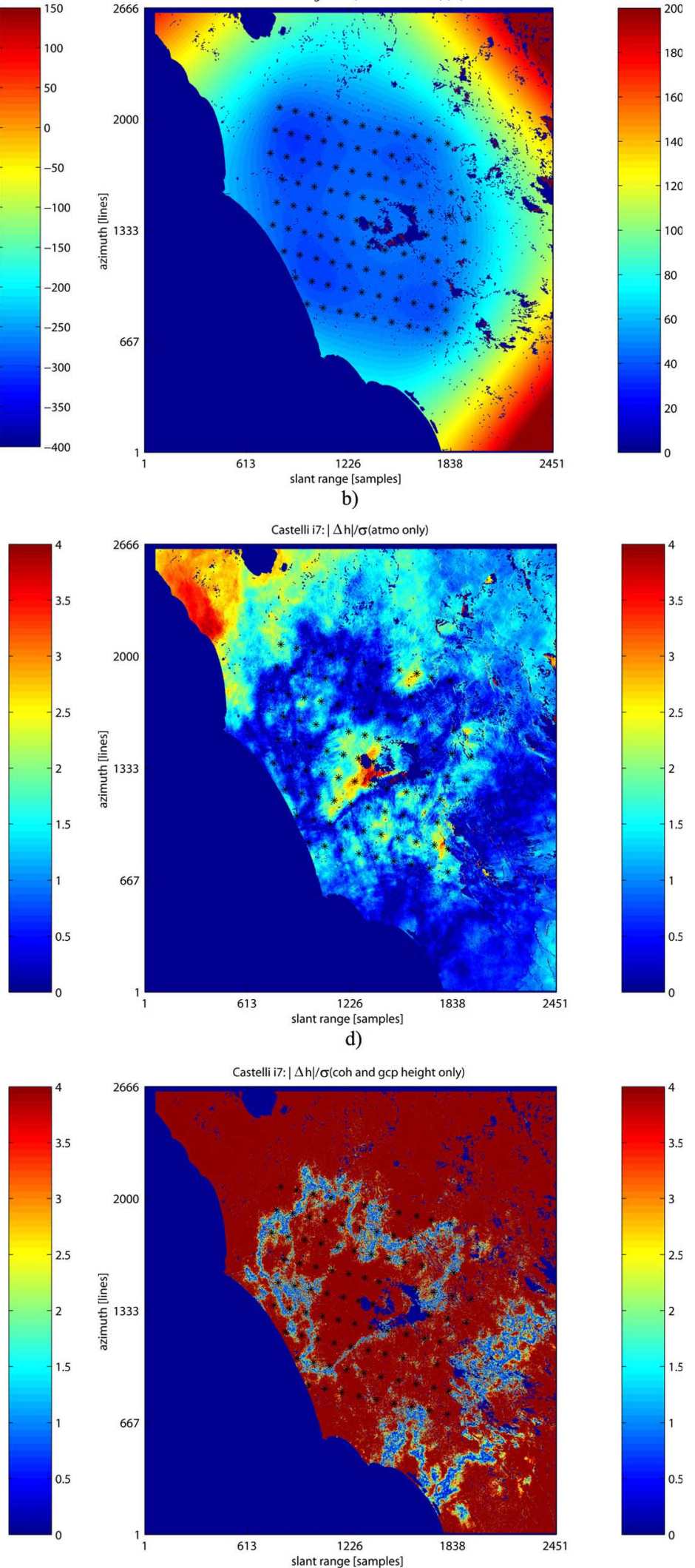

f)

Fig. 1. (a) Difference between I7 DEM and SRTM DEM. (b) Predicted height error standard deviation, accounting for all error sources. Height difference compared with SRTM DEM/predicted standard deviation accounting for (c) all error sources, (d) atmospheric propagation, (e) phase unwrapping errors, and (f) coherence and GCP height uncertainty. 


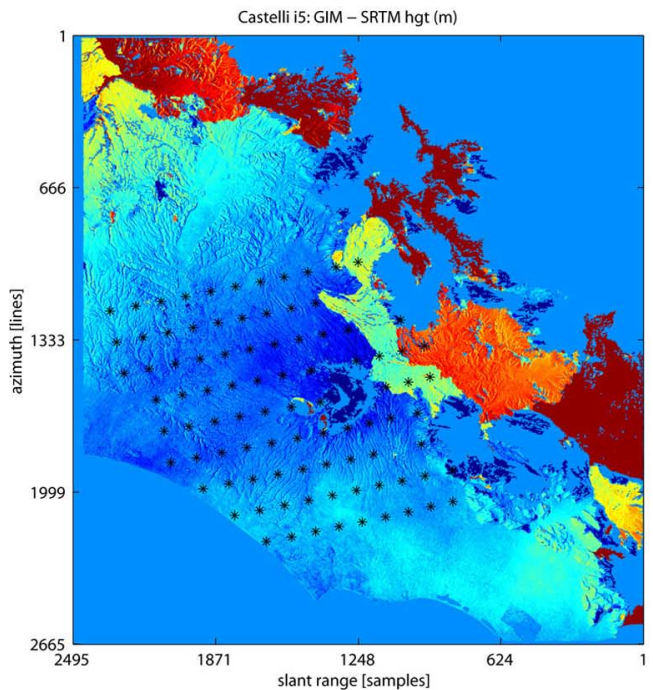

a)

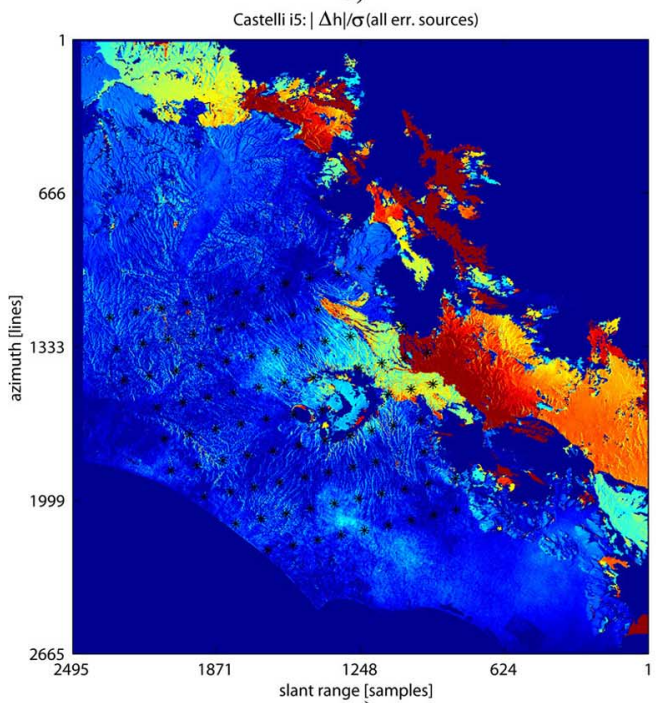

c)

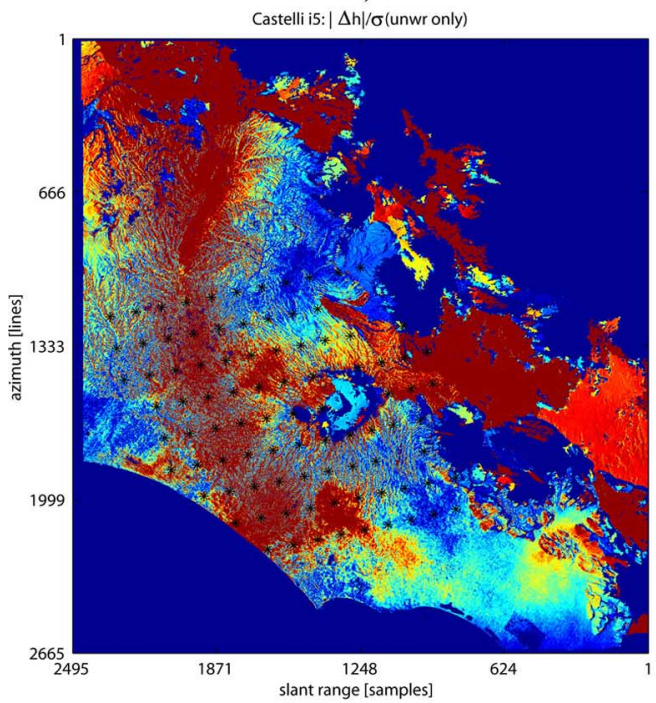

e)
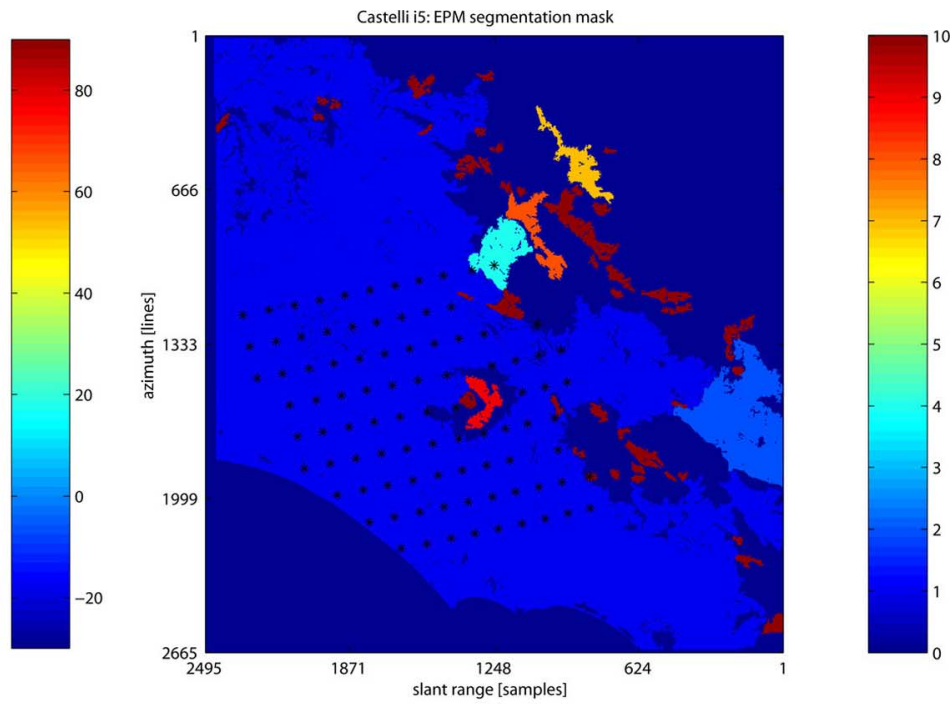

b)

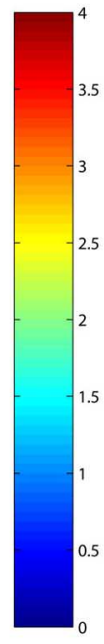

Castelli i5: $\mid \Delta \mathrm{h} / / \sigma($ atmo only)

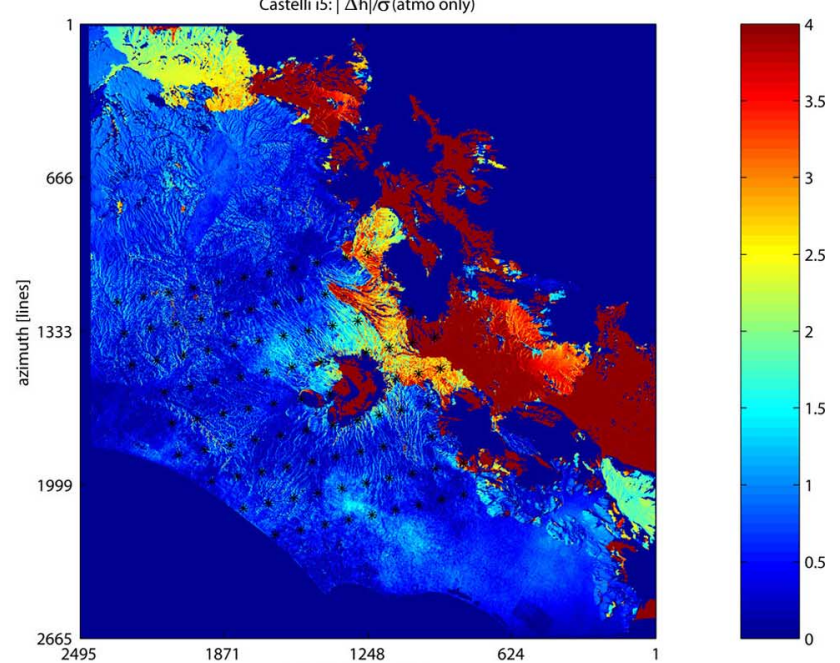

Castelli i5: $|\Delta h| / \sigma($ coh and gcp height only
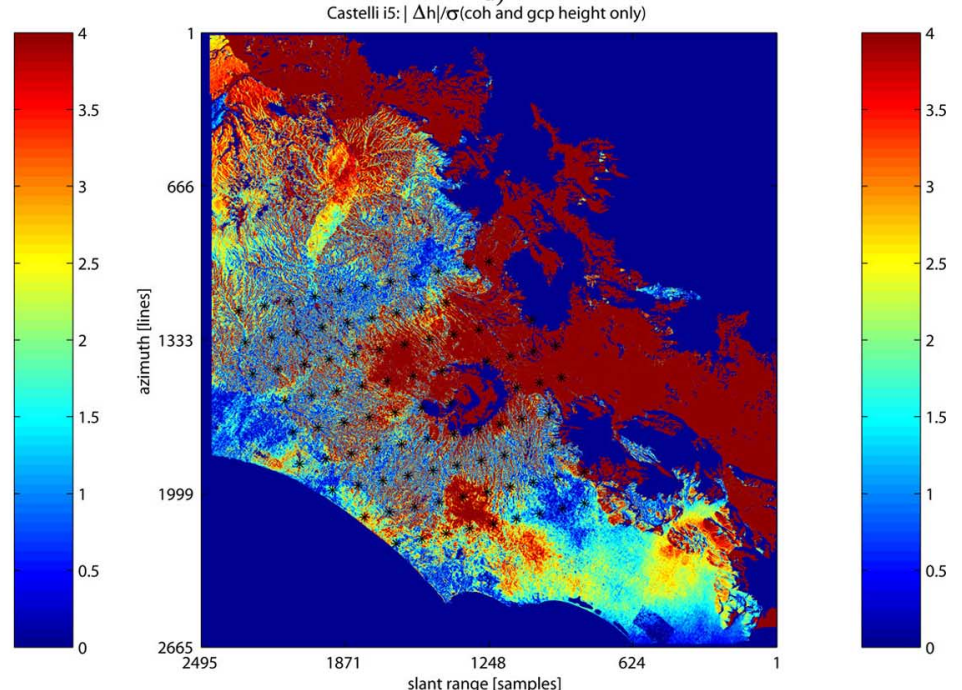

f)

Fig. 2. (a) Difference between I5 DEM and SRTM DEM. (b) Segmentation mask used by the phase unwrapping error model. Height difference compared with SRTM DEM/predicted standard deviation accounting for (c) all error sources, (d) atmospheric propagation, (e) phase unwrapping errors, and (f) coherence and GCP height uncertainty. 


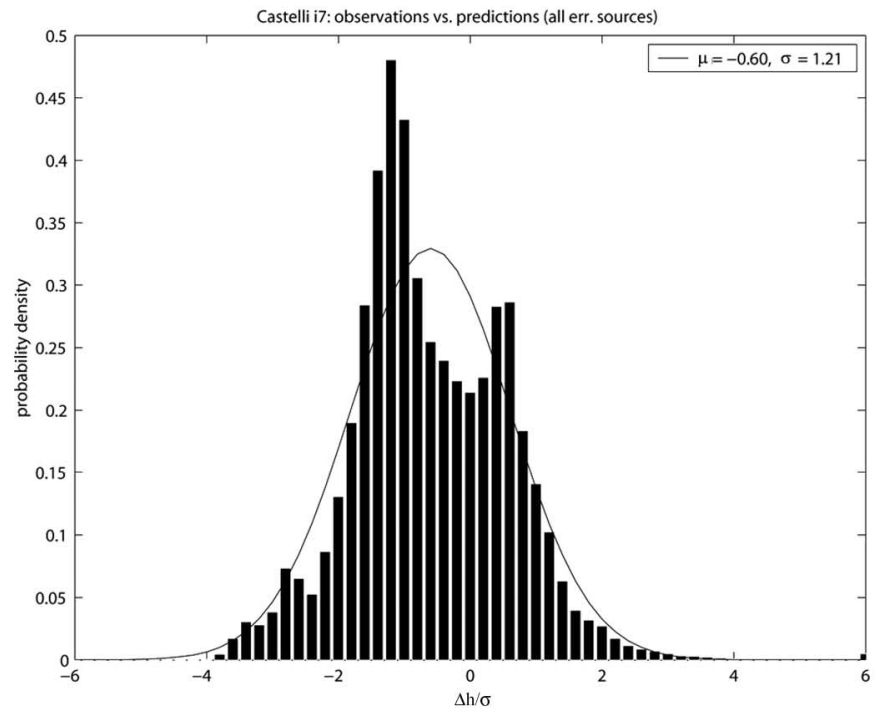

(a)

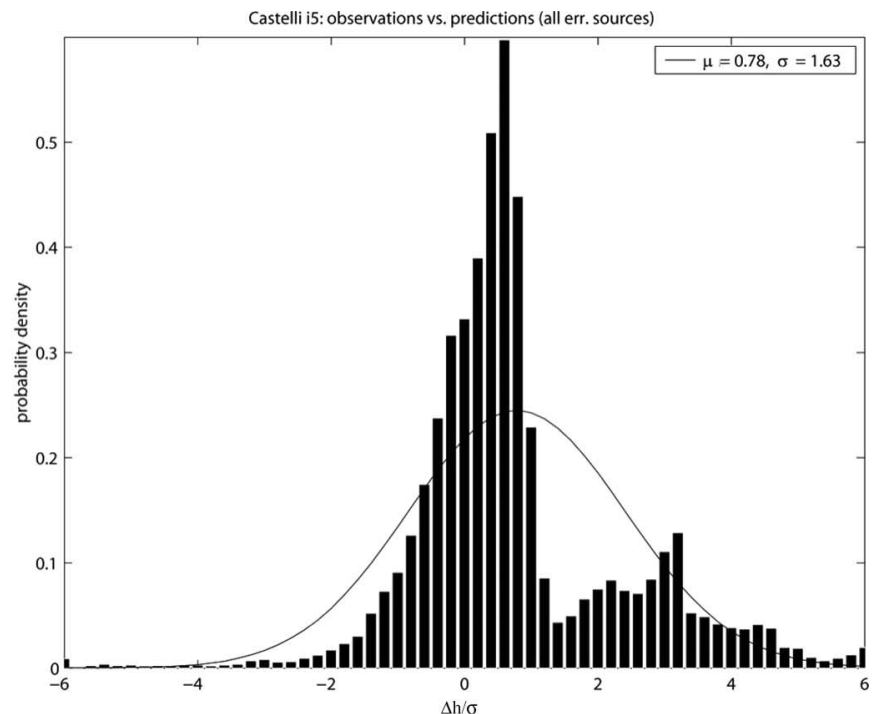

(b)

Fig. 3. Probability densities of the observed height difference compared with SRTM DEM/predicted standard deviation, respectively, for (a) I7 and (b) I5. The continuous curves represent the best fit Gaussian densities, with mean and variance reported in the top-right corner of each plot.

segments may be created in areas dense with poorly correlated pixels.

The predicted error standard deviation accounting for all error sources is shown in Fig. 2(c). In regions free from unwrapping errors, observations fall within two predicted standard deviations. By comparing Fig. 2(d)-(f), it can be noted that it is mainly the atmospheric error model which allows the correct order of magnitude of the differences to be explained. The phase unwrapping error model contributes by assigning an error of about one height of ambiguity to the disconnected segments it identifies. It is noted that the error model has no means of distinguishing single-cycle errors from multiple-cycle ones. For pixels lying in the main segment, where most of the GCPs are, the phase unwrapping error model assumes a maximum correlation with the GCP errors and, thus, a good calibration of this error source, from which a low predicted error follows.

The overall performance of the error prediction algorithm for I7 and I5 is summarized by the probability densities shown in Fig. 3. Ideally, the observed height differences normalized to the predicted standard deviation are expected to give a zero mean and a unity standard deviation. Fig. 3(a) refers to the I7 case and well approaches a Gaussian distribution with a standard deviation of 1.21. A negative bias is present, probably due to the fact that the tilt error along the image diagonal in Fig. 1(a) is not captured perfectly by the error models. This, in turn, might be due to the nonisotropic features of the observed atmospheric disturbance. In Fig. 3(b) instead, which refers to the I5 case, the distribution is not Gaussian, and the underestimates are due to the multiple-cycle phase unwrapping errors not predicted by our model.

\section{B. Application to Displacement Measurement}

The results of Section III-E were applied to predict the displacement measurement error using two ERS tandem pairs covering the province of Flevoland, The Netherlands, namely, frame 2547 from e1_23185/e2_03512 (21/22-12-95, $B_{\perp}=$ $173 \mathrm{~m})$ and e1_21181/e2_01508 (03/04-08-95, $\left.B_{\perp}=58 \mathrm{~m}\right)$. Over one day, the displacement in this region is expected to be close to zero, so that nonzero values in the velocity map essentially are measurement errors. GCPs extracted from a portion of the SRTM DEM of the region were used for the baseline calibration.

The double-difference technique was used to obtain a velocity map, as shown in Fig. 4(a) together with the GCPs used to calibrate each interferogram (represented with circles and asterisks, respectively). The observed deviations from the expected zero value are mainly due to atmospheric artifacts. In particular, in [9], it was concluded that the e1_21181/e2_01508 pair (referred to as FD1 in [9] and in the following) is affected by moisture fluctuations which cause path length variations up to half a wavelength $(2.8 \mathrm{~cm})$, whereas artefacts smaller than $7 \mathrm{~mm}$ are found in the e1_23185/e2_03512 pair (hereafter referred to as FD5).

A predicted error standard deviation was computed for each pixel using (24). Fig. 4(b)-(d) shows the ratio of the observed displacement to the predicted standard deviation, accounting, respectively, for all error sources, atmospheric propagation only, and coherence and GCP height uncertainty only. In Fig. 4(b), an overestimate in the left portion of the image is apparent. This is even more clear from the corresponding histogram of Fig. 5(a). The overestimate is partially due to the phase unwrapping error model, which incorrectly identifies a disjoint segment in one of the interferograms. However, elsewhere, this model also correctly flags unwrapping inconsistencies, as within the rectangular box on the top part of Fig. 4(a) and (b). As for the height error test cases, the atmospheric error model appears to add realism to the predictions compared with the case in which only coherence and GCP are accounted for. This is apparent from Fig. 4(c) and (d) and the histogram in Fig. 5(b). 


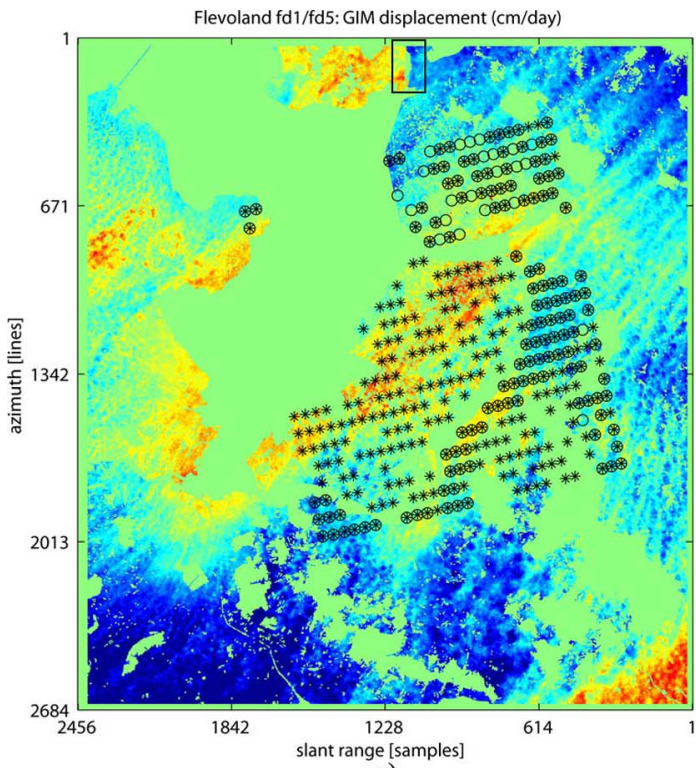

a)

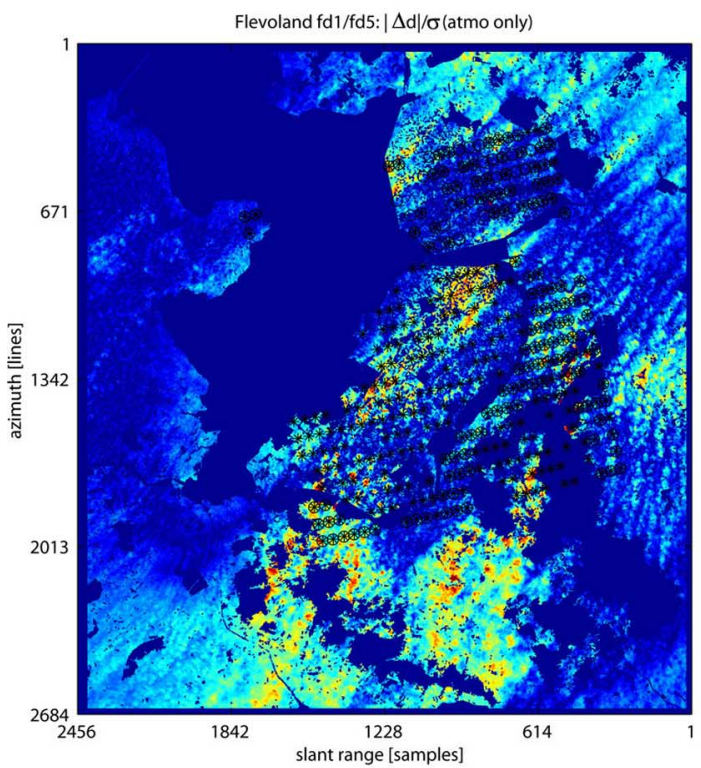

c)
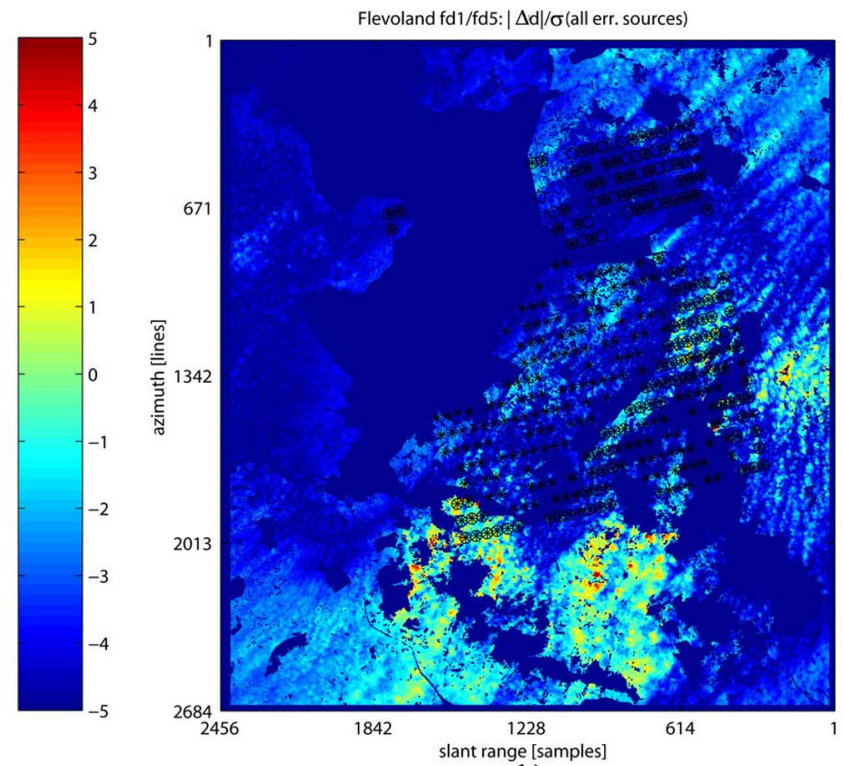

b)
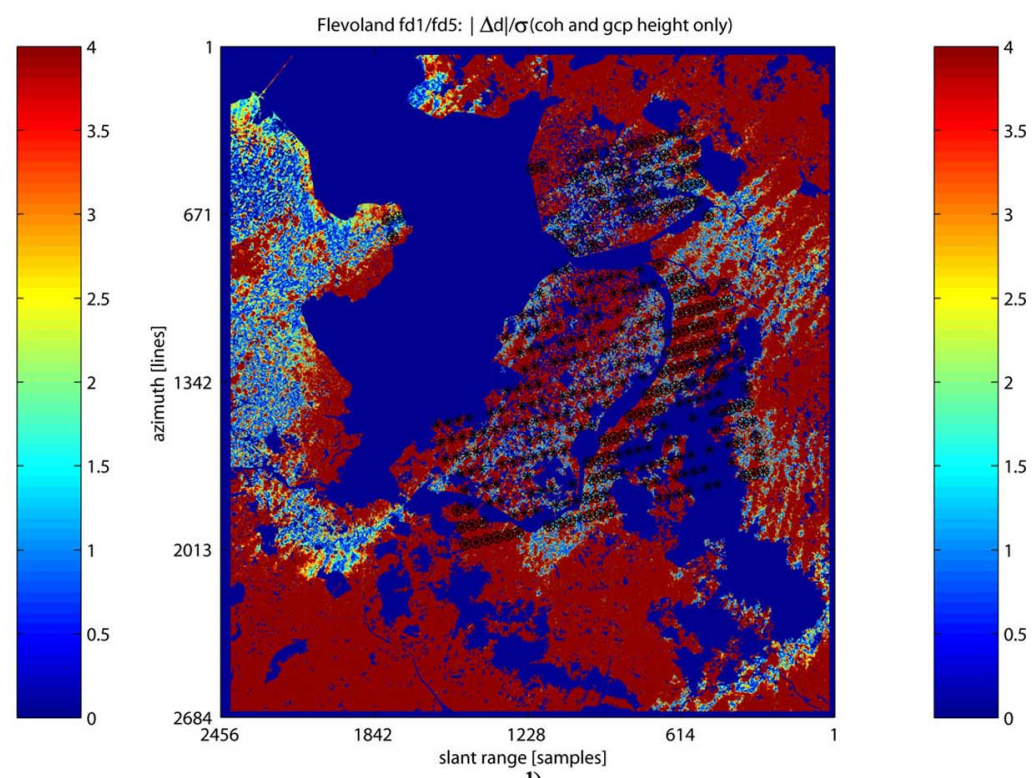

d)

Fig. 4. (a) FD1/FD5 measured displacement. Measured displacement/predicted standard deviation accounting for (b) all error sources, (c) atmospheric propagation, and (d) coherence and GCP height uncertainty.

\section{Assessment of the Results}

Height and displacement measurements from minimum interferometric data sets were considered. The proposed framework provided greatly improved error predictions compared with the standard approach in which only the local coherence value and the number of looks are considered.

Inclusion of an atmospheric error model, although just a standard one (i.e., not scene specific), proved to be very useful in predicting the correct magnitude of error standard deviations faraway from GCPs. It also provided a much more realistic prediction of the observed error magnitude compared with the conventional approach. The number and the entity of error underestimates and overestimates related to this error source are expected to improve using a scene-specific error model. Options for tuning the atmospheric model are discussed in Section V-A.
Inclusion of the unwrapping error model appeared useful to flag the presence of medium- to large-scale errors and did not contribute significantly to the overall prediction in the absence of apparent errors. However, only a part of the consistently unwrapped and disjoint segments were identified in our test cases. Furthermore, multiple- and single-cycle errors were handled in the same way by the model. Concerning the first issue, a further tuning of the model's parameters is expected to improve its performance, as further discussed in Section V-B.

A final remark concerns the presence of disconnected segments in the unwrapped interferometric phase. Typically, coefficients of first order and higher in the baseline calibration model, i.e., $\left(b_{2}, b_{3}, b_{4}\right)$ in (6), are estimated from the GCPs in the largest segment, whereas only a bias $b_{1}$ is estimated in each smaller segment, provided that at least one GCP is available [26]. This occurred in the Flevoland e1_23185/e2_03512 


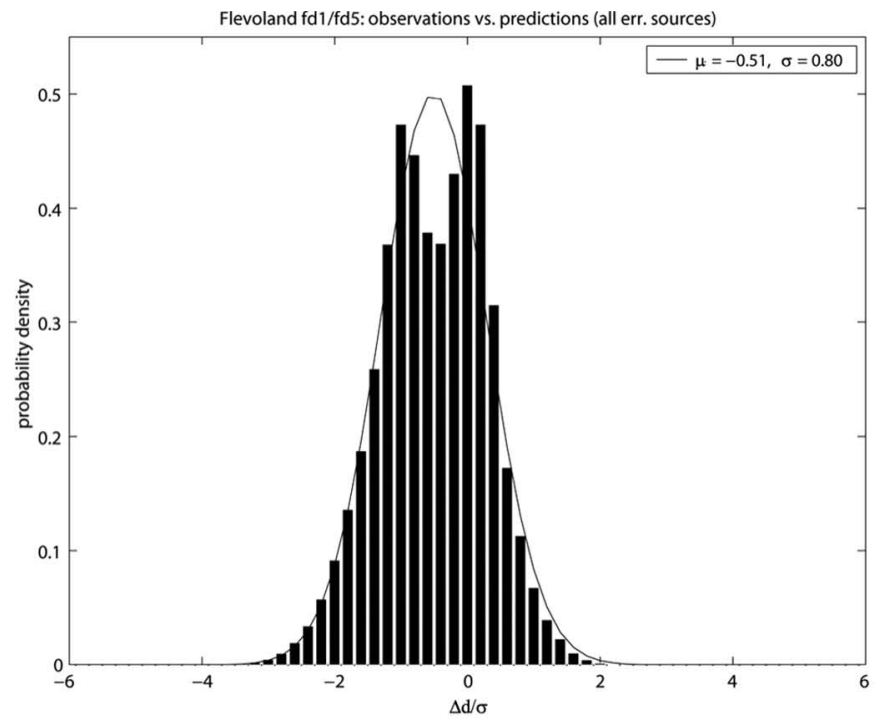

(a)

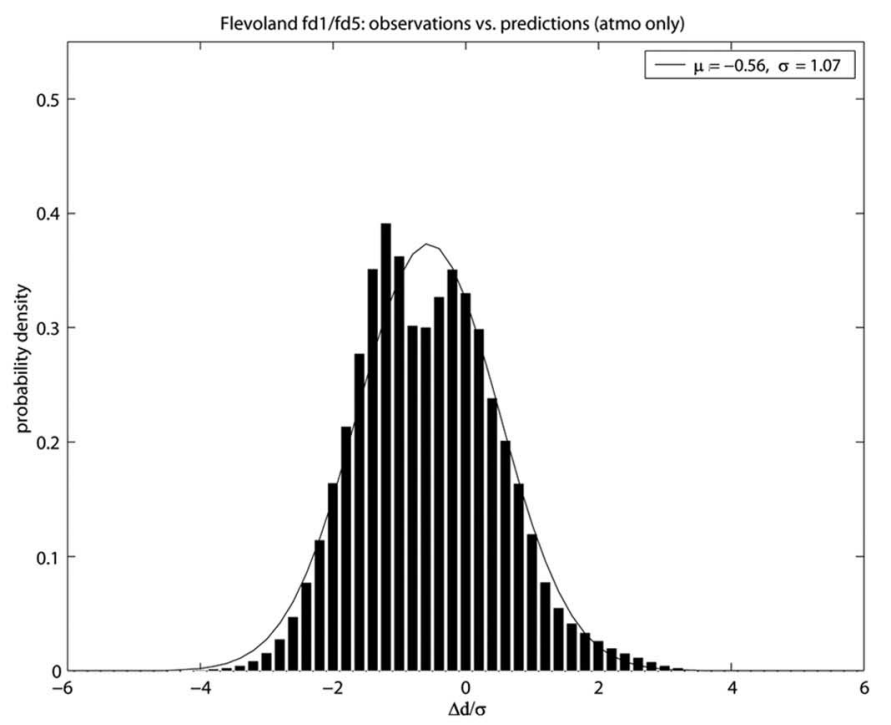

(b)

Fig. 5. Probability densities of the observed displacement/predicted standard deviation for FD1/FD5, respectively, (a) when all error sources are modeled and (b) when only atmospheric propagation is modeled. The continuous curves represent the best fit Gaussian densities, with mean and variance reported in the top-right corner of each plot.

interferogram, where only a subset of available GCPs was used [the asterisks in Fig. 4(a)]. Strictly speaking, (19) should be modified in each disconnected segment since the predicted variance will depend also on the GCPs therein, besides those in the largest one.

\section{DISCUSSION}

The outlined method produces a map of predicted error bars on a pixel-by-pixel basis. The prediction includes the major error sources and has proven to provide realistic estimates. This is a significant step forward compared with the methods relying solely on interferometric coherence.

Another aspect of error prediction is the correlation of errors on measurements in two pixels (often close together). The point is that even in areas with a large predicted error on a single-pixel basis, the measurements may locally provide results with good relative accuracy. The framework allows for the calculation of the correlation between errors in two pixels. The problem, however, is presentation since one, in principle, has to compute an error correlation map for each pixel in the image. Development of such error correlation products, e.g., useful for characterizing the terrain slope accuracies, is very important but considered outside the scope of this paper. It is also important to emphasize that the method is a framework, where the different elements can be improved individually in the future. In the remaining part of this section, shortcomings of some of the elements are described, along with suggestions for improvements.

\section{A. Tuning of the Tropospheric Delay Model}

The results of Section IV were obtained by using globally expected values for the atmospheric model parameters $P_{0}, h$, and $L$ in (9). Based on [5], different atmospheric states may approximately be described by variations of $P_{0}$ alone, which can reach up to an order of magnitude, whereas $h$ and $L$ may be considered fixed. Variations of $P_{0}$ translate directly into predicted error variances through (20). Acquisition-specific values for this parameter should therefore be used whenever available. These may be obtained from the SAR data itself if signals of nonatmospheric origin are known or negligible in a portion of the interferogram or from external systems.

Several techniques have been proved to provide zenith-delay measurements and could therefore potentially be exploited, particularly numerical weather models, microwave radiometers [29], [30], imaging spectrometers (e.g., MERIS [31]), and GPS. Preliminary experiments concerning the tuning of $P_{0}$ using GPS and microwave radiometer time series are reported in [32].

\section{B. Unwrapping Error Model Limitations}

The aim of the model in Section II-D is to account for medium- to large-scale phase unwrapping errors. These impact directly on the error budget as well as indirectly through the baseline calibration whenever a significant number of tie points are affected by phase unwrapping errors. The model is composed of two steps, namely, the identification of consistently unwrapped segments and the assignment of second-order error statistics based on this segmentation mask. In our opinion, this approach has the potential of flagging the majority of areas where medium- to large-scale errors might have occurred, whereas a good quantification of these errors still requires significant work.

The first open problem concerns the optimal selection of the parameters used to identify reliably unwrapped areas, particularly the residue density threshold and the erosion window size. As more conservative values are chosen (a lower threshold and a larger window size), more segments are identified, but at the same time, existing segments are typically further segmented, 
and the masked-out region grows. The values selected for this paper were verified not to be extreme, which means that they allow the identification of a part of the disconnected segments but, at the same time, generate few false alarms. A larger number of test cases than those considered so far should be analyzed however to determine the optimal combination of these parameters.

Second, in computing the covariance function, some oversimplifications are present. The first is to consider complete decorrelation between errors affecting pixels in different segments; the second is that the variance is assigned, assuming only single-cycle errors to occur. The former assumption is effective in confining the error prediction to where unwrapping inconsistencies are expected; however, if the segmentation mask is very conservative, several local false alarms may be generated due to the large number of masked-out pixels. The latter assumption is not trivial to overcome since knowledge of the number of erroneous cycles added by the unwrapper, if available, should be exploited by the unwrapping algorithm itself.

\section{Ionosphere}

It is a common practice to include compensation for ionospheric errors in the baseline calibration. Currently, orbit data for the ERS/ENVISAT satellites have accuracies on the order of 5-cm rms vertically and $15-\mathrm{cm}$ rms horizontally [33], [34]. The ionosphere, though, potentially causes a much larger slant range uncertainty. The question discussed next is whether it is reasonable to model ionospheric disturbances as baseline uncertainties.

A good review of the influence of ionosphere is given in [5]. The additional zenith delay caused by the ionosphere is

$$
\delta_{\text {iono }}^{\text {zenith }}=\frac{K}{f^{2}} \cdot \text { TEC } \quad K=-40.28 \mathrm{~m}^{3} \cdot \mathrm{s}^{-2}
$$

where TEC is the total electron content per square meter (column). Introducing the mapping function $1 / \cos \theta$ to the slant range geometry and the more practical TEC unit (TECU) being $10^{16}$ electrons $/ \mathrm{m}^{2}$ yields

$$
\frac{\partial \delta_{\text {iono }}}{\partial \text { TECU }} \approx-0.015 \mathrm{~m}^{3} \quad\left(\text { for C-band at } 23^{\circ}\right) .
$$

Typical TECU values vary from 0 at night to 20 during daytime in periods with minimum solar activity, increasing to 100 in periods with intense solar activity. For interferometry, it is not the TEC but the spatial variations of the differential TEC during the time span of the interferogram that cause the path length differences. Nevertheless, (28) indicates that even during periods with little solar activity, the ionosphere may give path length disturbances of the same order of magnitude as baseline uncertainties.

Due to the varying angle of incidence from near to far range, even a homogeneous electron layer will give a range phase ramp [10]. It is noted, though, that for the narrow $100-\mathrm{km}$ swath of the C-band ERS system, this effect is small, and consequently, only TEC variations (not a mean difference) cause phase errors.
TABLE I

Definitions of Four Test SCENES. START AND END Positions Were CHOSEN SO THAT NO SPATIAL INTERPOLATION IN THE GIMS IS

REQUIRED. IT IS NOT POSSIBLE TO AVOID TEMPORAL INTERPOLATION AS GIMS BEFORE Mid-2002 ARE PROVIDEd AT OdD Hours, Whereas GIMs After Are Provided at Even Hours. TRACK REFERS TO ERS IN THE 35-DAy REPEAT CYCLE

\begin{tabular}{l|rrrrr|r}
\hline \hline Site & UTC & lat. & lon. & lat. & lon. & track \\
\hline EUR-A & $20: 56$ & 35 & 20 & 55 & 15 & 458 \\
EUR-D & $08: 59$ & 55 & 30 & 35 & 25 & 422 \\
ARC-A & $00: 58$ & 60 & -45 & 80 & -75 & 217 \\
ARC-D & $14: 56$ & 80 & -30 & 60 & -60 & 397 \\
\hline \hline
\end{tabular}

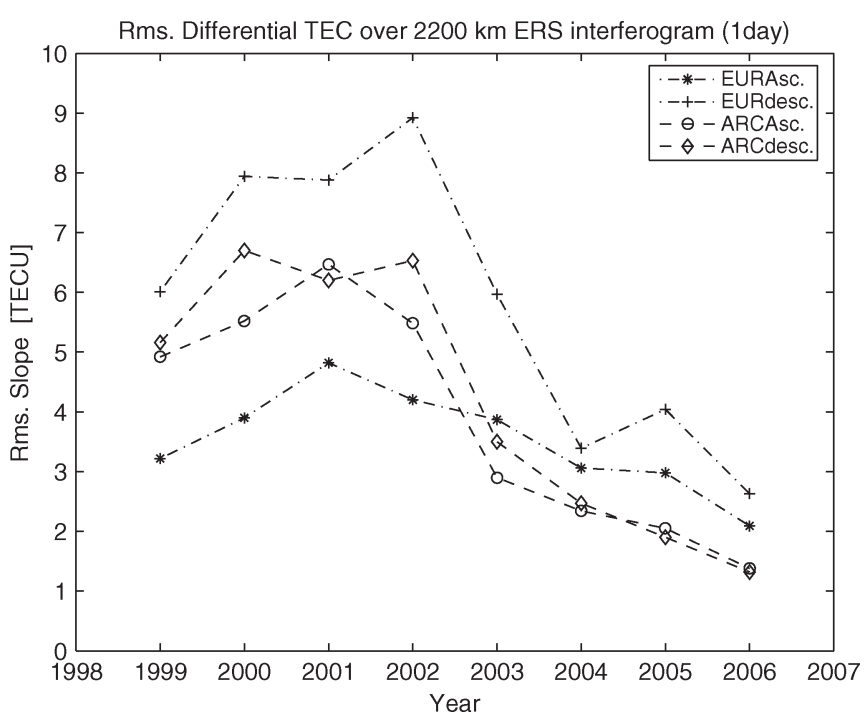

Fig. 6. Yearly rms values of differential TEC over 2200-km example scenes defined in Table I. Time separation: One day.

A good account of the different effects is given in [10]- a design study for an L-band SAR for TECU measurements. However, despite the fact that ionospheric effects on SAR interferometry have been studied thoroughly theoretically, few observations have actually been reported. A very convincing proof for short-scale effects causing streaks with low interferometric coherence has been given by Gray et al. [12]. The magnitude of the variations was about 0.1 TECU on a scale of about $1 \mathrm{~km}$. In [35], phase variations have been observed over a 3000-km strip, although the contributions of the baseline and ionospheric errors and of the proposed system drift error were not separated.

Recently, global ionospheric maps (GIMs) of TEC have been used in a sensitivity study for a future L-band single-pass interferometric SAR mission [11]. The 15-min average TECU values derived from about 100 GPS stations distributed globally, providing global maps with roughly $500-\mathrm{km}$ resolution, were used to estimate TEC at different times of the day and different locations.

In order to estimate the ionospheric effect on repeat-pass interferometric SAR, we used a similar approach based on the time series of GIMs with two-hourly TEC averages publicly available through the international GPS service network [36]. 


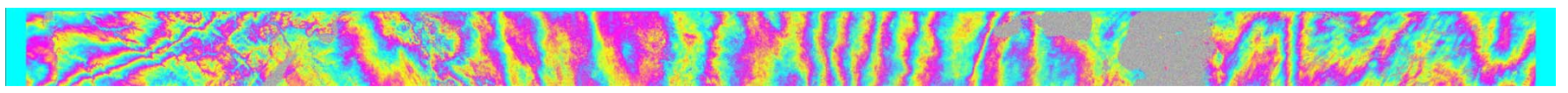

Fig. 7. Flattened interferogram from ERS-1, orbits 1014 and 1100, frames 927-1287, processed with Delft orbits, Gamma RS MSP, and internal software for interferogram formation.

Due to the rather poor spatial and temporal resolution of the GPS derived data, only differential trends over long (2200 km) strips are considered. Thus, the investigation gives a lower limit on ionospheric influence (provided that the GIMs have sufficient accuracy). Ascending and descending test scenes were defined both over Europe and over the Arctic (see Table I). For each possible SAR acquisition, the TEC trend was calculated as the difference between the TEC values at the two ends of the scene. Then, for a given time separation of SAR acquisitions, the differential trend, which is denoted here as slope, was calculated.

The first full year available is 1999. For each year, data are available from at least five different data centers. Initially, slope averages for year 2000 were calculated for time separations of 1 and 35 days for each of the four scenes and for all data centers. Since the Center for Orbit Determination in Europe (CODE), Switzerland, data generally provided median results for the EUR sites and the Jet Propulsion Laboratory provided median results for the ARC sites (but with CODE results very similar), we chose to continue solely with the CODE data.

The yearly slope rms for a one-day time separation is shown in Fig. 6. The solar activity maximum around 2001 is clearly seen to give larger slope variations. In addition, a clear difference between descending (local time 1050) and ascending (local time 2210) tracks is seen for the European scenes as opposed to the Arctic scenes where slope rms is similar for the ascending and descending orbit tracks.

The 35-day time-separation results were more noisy due to the reduced number of samples per year but showed a similar behavior, which is just scaled by a factor of 1.25.

The conclusion is that for C-band, narrow swath, and standard scenes, it is reasonable to pool ionosphere and baseline. For L-band or long strips, it seems more reasonable to include the ionosphere as an error source using an approach similar to the one used for the troposphere. Indications on the associated power law and how it can be tuned are given in [11].

\section{System Clock Drift}

In [35], large along-track phase variations that were not likely to originate from tropo-/ionosphere were reported. Drift of the radar local oscillator was proposed as an explanation. A reprocessing by using Delft orbits, the Modular SAR Processor from Gamma Remote Sensing for SLC generation, and internal software for interferogram formation and flattening showed a much less pronounced phase variation (Fig. 7). The resulting interferogram is similar to the one reported in [37, p. C-91]. The reprocessed interferogram still exhibits large phase variations/ trends but not so large for an unusual combination of tropospheric, ionospheric, and baseline errors to be ruled out as an explanation. According to Fletcher [37, p. C-90], "Several attempts to find more examples of the 'clock artefact,' particu- larly for ERS-2, failed...” Thus, we suggest to ignore the system clock drift, at least for short strips.

\section{CONCLUSION}

A framework for providing a priori error estimates for satellite interferometric radar elevation and displacement products was developed, demonstrated, and discussed. The method accounts for baseline, atmospheric, and phase unwrapping errors. Compared with an approach relying solely on interferometric coherence, the general error trends are better captured, and the error estimates are significantly more realistic. Presently, the framework uses a troposphere model with fixed parameters, although additional information on the atmospheric state, if available, could be used to tune the model. The unwrapping error modeling approach has, in our opinion, the potential of flagging, and partially of quantifying, the medium- to largescale errors. However, a dedicated study aimed at fine-tuning the model's parameters is required in order to find the best compromise between the number of detected errors and that of false alarms.

\section{APPENDIX \\ STRUCTURE Function For Tropospheric DELAY}

In [16], the following structure function for the zenith tropospheric delay is proposed:

$$
\begin{aligned}
R^{\prime} & =\frac{R}{h} \quad u=\pi \frac{R}{h} \\
D(R) & =P_{0} C_{0}\left[\frac{C_{1} I_{1}\left(R^{\prime}\right) R^{2 / 3}}{1+\left(\frac{R}{L}\right)^{2 / 3}}+C_{2} I_{2}\left(R^{\prime}\right) R^{5 / 3}\right] \\
C_{0} & =(\lambda / 4 \pi)^{2} \\
C_{1} & =4 f_{0}^{8 / 3} \pi^{2 / 3} h \\
C_{2} & =4 f_{0}^{8 / 3} \pi^{5 / 3} \\
I_{1}\left(R^{\prime}\right) & = \begin{cases}\frac{3}{4} u^{4 / 3}-\frac{1}{10} u^{10 / 3}, & R^{\prime} \leq A_{1} \\
C_{3}-\frac{3}{4} u^{-2 / 3}, & R^{\prime}>A_{1}\end{cases} \\
I_{2}\left(R^{\prime}\right) & = \begin{cases}C_{4}-3 u^{1 / 3}+\frac{1}{7} u^{7 / 3}, & R^{\prime} \leq A_{2} \\
\frac{3}{10} u^{-5 / 3}, & R^{\prime}>A_{2}\end{cases}
\end{aligned}
$$

where $P_{0}=9 \mathrm{~m}, L=2133 \mathrm{~km}, h=3 \mathrm{~km}, f_{0}=1 \mathrm{~km}^{-1}$, $A_{1}=0.472, A_{2}=0.466, C_{3}=1.473$, and $C_{4}=3.218$. The magnitude of the disturbance is tuned through $P_{0}$. The transition between the two turbulence regimes is determined by $h$.

\section{ACKNOWLEDGMENT}

The authors would like to thank the European Space Agency for providing the images through AO3-114 and Category-1 Project ID 1396. 


\section{REFERENCES}

[1] A. Ferretti, C. Prati, and F. Rocca, "Permanent scatterers in SAR interferometry," IEEE Trans. Geosci. Remote Sens., vol. 39, no. 1, pp. 8-20, Jan. 2001.

[2] I. Joughin, "Ice-sheet velocity mapping: A combined interferometric and speckle-tracking approach," Ann. Glaciol., vol. 34, no. 1, pp. 195-201, Jan. 2002.

[3] I. Joughin, R. Kwok, and M. Fahnestock, "Estimation of ice-sheet motion using satellite radar interferometry: Method and error analysis with application to Humboldt Glacier, Greenland," J. Glaciol., vol. 42, no. 142, pp. 564-575, 1996.

[4] J. J. Mohr, N. Reeh, and S. N. Madsen, "Accuracy of three-dimensional glacier surface velocities derived from radar interferometry and icesounding radar measurements," J. Glaciol., vol. 49, no. 165, pp. 210-222, 2003.

[5] R. Hanssen, Radar Interferometry: Data Interpretation and Error Analysis. Dordrecht, The Netherlands: Kluwer, 2001.

[6] R. M. Goldstein, "Atmospheric limitations to repeat-track radar interferometry," Geophys. Res. Lett., vol. 22, no. 18, pp. 2517-2520, Sep. 1995.

[7] H. Tarayre and D. Massonnet, "Atmospheric propagation heterogeneities revealed by ERS-1," Geophys. Res. Lett., vol. 23, no. 9, pp. 989-992, May 1996.

[8] H. A. Zebker, P. A. Rosen, and S. Hensley, "Atmospheric effects in interferometric synthetic aperture radar surface deformation and topographic maps," J. Geophys. Res., vol. 102, no. B4, pp. 7547-7563, Apr. 1997.

[9] R. Hanssen, "Atmospheric heterogeneities in ERS tandem SAR interferometry," Delft Univ., Delft, The Netherlands, DEOS Report 98.1, 1998.

[10] F. Meyer, R. Bamler, N. Jakowski, and T. Fritz, "The potential of lowfrequency SAR systems for mapping ionospheric TEC distributions," IEEE Geosci. Remote Sens. Lett., vol. 3, no. 4, pp. 560-564, Oct. 2006.

[11] E. Chapin, S. F. Chan, B. D. Chapman, C. W. Chen, J. M. Martin, T. R. Michel, R. J. Muellerschoen, X. Pi, and P. A. Rosen, "Impact of the ionosphere on an L-band space based radar," in Proc. IEEE Conf. Radar, Apr. 24-27, 2006, pp. 51-58.

[12] L. Gray, K. E. Mattar, and G. Sofko, "Influence of ionospheric electron density fluctuations on satellite radar interferometry," Geophys. Res. Lett., vol. 27, no. 10, pp. 1451-1454, May 2000.

[13] T. R. Emardson, M. Simons, and F. H. Webb, "Neutral atmospheric delay in interferometric synthetic aperture radar applications: Statistical description and mitigation," J. Geophys. Res., vol. 108, no. B5, pp. 2231-2238, May 2003.

[14] R. N. Treuhaft and G. E. Lanyi, "The effect of the dynamic wet troposphere on radio interferometric measurements," Radio Sci., vol. 22, no. 2, pp. 251-265, Mar./Apr. 1987.

[15] S. Williams, Y. Bock, and P. Fang, "Integrated satellite interferometry: Tropospheric noise, GPS estimates and implications for interferometric synthetic aperture radar products," J. Geophys. Res., vol. 103, no. B11, pp. $27051-27067,1998$.

[16] J. P. Merryman Boncori and J. J. Mohr, "A tunable closed form model for the structure function of tropospheric delay," IEEE Geosci. Remote Sens. Lett., vol. 5, no. 2, pp. 222-226, Apr. 2008.

[17] A. A. Stotskii, "Concerning the fluctuating characteristics of Earth's troposphere," Radiophys. Quantum Electron., vol. 16, no. 5, pp. 620-622, 1973. (Translated from Russian by Springer New York).

[18] F. A. Gifford, "A similarity theory of the tropospheric turbulence energy spectrum," J. Atmos. Sci., vol. 45, no. 8, pp. 1370-1379, 1988.

[19] E. Rodriguez and J. M. Martin, "Theory and design of interferometric synthetic aperture radars," Proc. Inst. Electr. Eng.-F, Radar Signal Process., vol. 139, no. 2, pp. 147-159, Apr. 1992.

[20] C. Werner, U. Wegmüller, and T. Strozzi, "Processing strategies for phase unwrapping for INSAR applications," in Proc. EUSAR, Jun. 4-6, 2002, pp. 353-355.

[21] O. Hellwich, "SAR phase unwrapping: Implications of terrain shape and smoothing," in Proc. EUSAR, May 25-27, 1998, pp. 51-56.

[22] M. Eineder, "Efficient simulation of SAR interferograms of large areas and of rugged terrain," IEEE Trans. Geosci. Remote Sens., vol. 41, no. 6, pp. 1415-1427, Jun. 2003.

[23] S. Suchandt and M. Eineder, "Experiences with SRTM/X-SAR phase unwrapping using minimum cost flow method," in Proc. IEEE Int. Geosci. Remote Sens. Symp., Jul. 21-25, 2003, vol. 7, pp. 4380-4382.

[24] C. W. Chen and H. A. Zebker, "Network approaches to twodimensional phase unwrapping: Intractability and two new algorithms," J. Opt. Soc. Amer. A, Opt. Image Sci., vol. 17, no. 3, pp. 401-414, Mar. 2000.

[25] C. W. Chen and H. A. Zebker, "Two dimensional phase unwrapping with use of statistical models for cost functions in nonlinear optimiza- tion," J. Opt. Soc. Amer. A, Opt. Image Sci., vol. 18, no. 2, pp. 338-351, Feb. 2001.

[26] M. Hubig, S. Suchandt, and M. Eineder, "Automatic correction of baseline and phase unwrapping errors in SAR interferograms," in Proc. EUSAR, May 23-25, 2000, pp. 131-134.

[27] L. Galli, "A new approach based on network theory to locate phase unwrapping unreliable results," in Proc. IEEE Int. Geosci. Remote Sens. Symp., Jul. 9-13, 2001, vol. 1, pp. 118-120.

[28] D. Massonnet and K. L. Feigl, "Discrimination of geophysical phenomena in satellite radar interferograms," Geophys. Res. Lett., vol. 22, no. 12, pp. 1537-1540, Jun. 1995.

[29] K. Snajdrova, J. Boehm, P. Willis, R. Haas, and H. Schuh, "Multitechnique comparison of tropospheric zenith delays derived during the CONT02 campaign," J. Geod., vol. 79, no. 10/11, pp. 613-623, Jan. 2006.

[30] A. E. Niell, A. J. Coster, F. S. Solheim, V. B. Mendes, P. C. Toor, R. B. Langley, and C. A. Upham, "Comparison of measurements of atmospheric wet delay by radiosonde, water vapor radiometer, GPS, and VLBI," J. Atmos. Ocean. Technol., vol. 18, no. 6, pp. 830-850, Jun. 2001.

[31] Z. Li, E. J. Fielding, and J. P. Muller, "Interferometric synthetic aperture radar atmospheric correction: Medium resolution imaging spectrometer and advanced synthetic aperture radar integration," Geophys. Res. Lett., vol. 33, no. 6, p. L06 816, 2006.

[32] D. N. Moissev, R. F. Hanssen, and F. J. van Leijen, "Water vapor observations with SAR, microwave radiometer and GPS: Comparison of scaling characteristics," in Proc. ERAD, Nov. 18-22, 2002, pp. 190-194.

[33] E. Doornbos and R. Scharroo, "Improved ERS and ENVISAT precise orbit determination," presented at the Proc. ENVISAT ERS Symp., pp. 401408, Apr. 2005, ESA SP-572.

[34] R. Zandbergen, M. Otten, P. L. Righetti, D. Kuijper, and J. M. Dow, "Routine operational and high-precision orbit determination of ENVISAT," Adv. Space Res., vol. 31, no. 8, pp. 1953-1958, 2003.

[35] D. Massonnet and H. Vadon, "ERS-1 internal clock drift measured by interferometry," IEEE Trans. Geosci. Remote Sens., vol. 33, no. 2, pp. 401-408, Mar. 1995.

[36] J. M. Dow, R. E. Neilan, and G. Gendt, "The international GPS service (IGS): Celebrating the 10th anniversary and looking to the next decade," Adv. Space Res., vol. 36, no. 3, pp. 320-326, 2005.

[37] InSAR Principles: Guidelines for SAR Interferometry Processing and Interpretation, K. Fletcher, Ed. Noordwijk, The Netherlands: ESA Publications, Feb. 2007. TM-19.

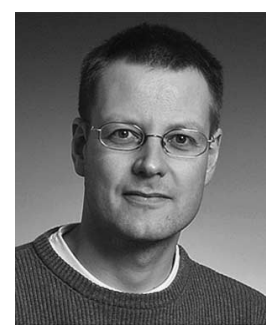

Johan Jacob Mohr received the M.Sc. degree in electrical engineering and the Ph.D. degree from the Technical University of Denmark, Kgs. Lyngby, Denmark, in 1992 and 1997, respectively.

Since then, he has been working with the ØrstedDTU, Technical University of Denmark, where he is currently an Associate Professor. His main research interests include methods and principles relating to satellite and airborne SAR interferometry, particularly as applied to glaciological studies. This includes quantitative estimation of errors in interfer-

ometric radar products.

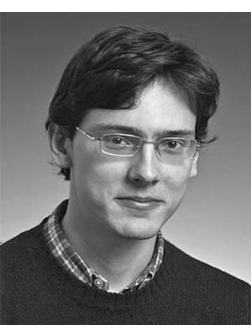

John Peter Merryman Boncori was born in London, U.K., on December 2, 1980. He received the "Laurea" degree in communications engineering (summa cum laude) from the University of Rome "Tor Vergata," Rome, Italy, in 2003, carrying out his thesis at ESA/ESRIN on encoding SAR transponder signal processing, and the Geoinformation Ph.D. degree from the University of Rome "Tor Vergata," in 2007 .

Since January 2006, he has been with the Department of Electromagnetic Systems, Technical University of Denmark, Kgs. Lyngby, Denmark, as a visiting Ph.D. student and currently as a Postdoctoral Fellow with DTU Space, working on error modeling and correction techniques for SAR interferometry. 